\title{
Pacific
}

Journal of

Mathematics

\section{ON THE CENTER OF FUSION CATEGORIES}

Alain Bruguières AND Alexis Virelizier

Volume $264 \quad$ No. 1 


\title{
ON THE CENTER OF FUSION CATEGORIES
}

\author{
Alain BRUguiÈres AND AleXis ViRelizier
}

\begin{abstract}
Müger proved in 2003 that the center of a spherical fusion category $\mathscr{C}$ of nonzero dimension over an algebraically closed field is a modular fusion category whose dimension is the square of that of $\mathscr{G}$. We generalize this theorem to a pivotal fusion category $\mathscr{b}$ over an arbitrary commutative ring $\mathbb{k}$, without any condition on the dimension of the category. (In this generalized setting, modularity is understood as 2-modularity in the sense of Lyubashenko.) Our proof is based on an explicit description of the Hopf algebra structure of the coend of the center of $\mathscr{C}$. Moreover we show that the dimension of $\mathscr{C}$ is invertible in $\mathbb{k}$ if and only if any object of the center of $\mathscr{b}$ is a retract of a "free" half-braiding. As a consequence, if $k$ is a field, then the center of $\mathscr{C}$ is semisimple (as an abelian category) if and only if the dimension of $\mathscr{C}$ is nonzero. If in addition $\mathbb{k}$ is algebraically closed, then this condition implies that the center is a fusion category, so that we recover Müger's result.
\end{abstract}

$\begin{array}{lr}\text { Introduction } & 1 \\ \text { 1. Pivotal and fusion categories } & 3 \\ \text { 2. Main results } & 9 \\ \text { 3. Modular categories } & 12 \\ \text { 4. Proofs } & 16 \\ \text { References } & 29\end{array}$

\section{Introduction}

Given a monoidal category $\mathscr{C}$, Joyal and Street [1991], Drinfeld (unpublished), and Majid [1991] defined a braided category $\mathscr{E}(\mathscr{C})$, called the center of $\mathscr{C}$, whose objects are half-braidings of $\mathscr{C}$. Müger [2003] showed that the center $\mathscr{L}(\mathscr{C})$ of a spherical fusion category $\mathscr{C}$ of nonzero dimension over an algebraically closed field $\mathbb{k}$ is a modular fusion category, and that the dimension of $\mathscr{E}(\mathscr{C})$ is the square of that of $\mathscr{C}$. Müger's proof of this remarkable result relies on algebraic constructions due to Ocneanu (such as the "tube" algebra) and involves the construction of a

MSC2010: 18D10, 16T05, 18C20.

Keywords: categorical center, fusion categories, Hopf monads, modularity. 
weak monoidal Morita equivalence between $\mathscr{L}(\mathscr{C})$ and $\mathscr{b} \otimes \mathscr{C}^{\mathrm{op}}$. The modularity of the center is of special interest in three-dimensional quantum topology, since spherical fusion categories and modular categories are respectively the algebraic input for the construction of the Turaev-Viro/Barrett-Westbury invariant and of the Reshetikhin-Turaev invariant. Indeed it has been shown recently in [Turaev and Virelizier 2010] (see also [Balsam 2010]) that, under the hypotheses of Müger's theorem, the Barrett-Westbury generalization of the Turaev-Viro invariant for $\mathscr{b}$ is equal to the Reshetikhin-Turaev invariant for $\mathscr{L}(\mathscr{C})$.

In this paper, we generalize Müger's theorem to pivotal fusion categories over an arbitrary commutative ring. More precisely, given a pivotal fusion category $\mathscr{b}$ over a commutative ring $\mathbb{k}$, we prove the following:

(i) the center $\mathscr{L}(\mathscr{C})$ of $\mathscr{C}$ is always modular (but not necessarily semisimple) and has dimension $\operatorname{dim}(\mathscr{C})^{2}$;

(ii) the scalar $\operatorname{dim}(\mathscr{C})$ is invertible in $\mathbb{k}$ if and only if every half braiding is a retract of a so-called free half braiding;

(iii) if $\mathbb{k}$ is a field, then $\mathscr{L}(\mathscr{C})$ is abelian semisimple if and only if $\operatorname{dim}(\mathscr{C}) \neq 0$;

(iv) if $\mathbb{k}$ is an algebraically closed field, then $\mathscr{L}(\mathscr{C})$ is fusion if and only if $\operatorname{dim}(\mathscr{C}) \neq 0$.

Our proof is different from that of Müger. It relies on the principle that if a braided category $\mathscr{B}$ has a coend, then all the relevant information about $\mathscr{B}$ is encoded in its coend, which is a universal Hopf algebra sitting in $\mathscr{B}$ and endowed with a canonical Hopf algebra pairing. For instance, modularity means that the canonical pairing is nondegenerate, and the dimension of $\mathscr{B}$ is that of its coend. In particular we do not need to introduce an auxiliary category.

The center $\mathscr{L}(\mathscr{C})$ of a pivotal fusion category $\mathscr{C}$ always has a coend. We provide a complete and explicit description of the Hopf algebra structure of this coend, which enables us to exhibit an integral for the coend and an "inverse" to the pairing. Our proofs are based on a "handleslide" property for pivotal fusion categories.

A general description of the coend of the center of a rigid category $\mathscr{b}$, together with its structural morphisms, was given in [Bruguières and Virelizier 2012]. It is an application of the theory of Hopf monads, and in particular, of the notion of double of a Hopf monad, which generalizes the Drinfeld double of a Hopf algebra. It is based on the fact that $\mathscr{L}(\mathscr{C})$ is the category of modules over a certain quasitriangular Hopf monad $Z$ on $\mathscr{C}\left(\operatorname{generalizing}\right.$ the braided equivalence $\mathscr{L}\left(\bmod _{H}\right) \simeq \bmod _{D(H)}$ between the center of the category of modules over a finite-dimensional Hopf algebra $H$ and the category of modules over the Drinfeld double $D(H)$ of $H$ ). It turns out that, when $\mathscr{C}$ is a fusion category, we can make this description very explicit and in particular, we can depict the structural morphisms of the coend by means of a graphical formalism for fusion categories. 
Part of the results of this paper were announced (without proofs) in [Bruguières and Virelizier 2008], where they were used to define and compute a 3-manifolds invariant of Reshetikhin-Turaev type associated with the center of $\mathscr{C}$, even when the dimension of $\mathscr{C}$ is not invertible.

Organization of the text. In Section 1, we recall definitions, notations and basic results concerning pivotal and fusion categories over a commutative ring. A graphical formalism for representing morphisms in fusion categories is provided. In Section 2, we state the main results of this paper, that is, the description of the coend of the center of a pivotal fusion category and its structural morphisms, the modularity of the center of such a category, its dimension, and a semisimplicity criterion. Section 3 is devoted to coends, Hopf algebras in braided categories, and modular categories. Section 4 contains the proofs of the main results.

\section{Pivotal and fusion categories}

Monoidal categories are assumed to be strict. This does not lead to any loss of generality, since, in view of Mac Lane's coherence theorem for monoidal categories (see [Mac Lane 1998]), all definitions and statements remain valid for nonstrict monoidal categories after insertion of the suitable canonical isomorphisms.

1A. Rigid categories. Let $\mathscr{C}=(\mathscr{C}, \otimes, \mathbb{1})$ be a monoidal category. A left dual of an object $X$ of $\mathscr{C}$ is an object ${ }^{\vee} X$ of $\mathscr{C}$ together with morphisms ev ${ }^{\vee}{ }^{\vee} X \otimes X \rightarrow \mathbb{1}$ and $\operatorname{coev}_{X}: \mathbb{1} \rightarrow X \otimes{ }^{\vee} X$ such that

$$
\left(\operatorname{id}_{X} \otimes \mathrm{ev}_{X}\right)\left(\operatorname{coev}_{X} \otimes \mathrm{id}_{X}\right)=\mathrm{id}_{X} \quad \text { and } \quad\left(\mathrm{ev}_{X} \otimes \mathrm{id}_{X}\right)\left(\mathrm{id}_{v_{X}} \otimes \operatorname{coev}_{X}\right)=\mathrm{id}_{X} .
$$

Similarly a right dual of $X$ is an object $X^{\vee}$ with morphisms $\widetilde{\mathrm{ev}}_{X}: X \otimes X^{\vee} \rightarrow \mathbb{1}$ and $\widetilde{\operatorname{cov}}_{X}: \mathbb{1} \rightarrow X^{\vee} \otimes X$ such that

$$
\left(\widetilde{\mathrm{ev}}_{X} \otimes \mathrm{id}_{X}\right)\left(\mathrm{id}_{X} \otimes \widetilde{\operatorname{cov}}_{X}\right)=\mathrm{id}_{X} \quad \text { and } \quad\left(\mathrm{id}_{X} \vee \widetilde{\operatorname{ev}}_{X}\right)\left(\widetilde{\operatorname{cov}}_{X} \otimes \mathrm{id}_{X} \vee\right)=\mathrm{id}_{X^{\vee}}
$$

The left and right duals of an object, if they exist, are unique up to an isomorphism (preserving the (co)evaluation morphisms).

A monoidal category $\mathscr{b}$ is rigid (or autonomous) if every object of $\mathscr{C}$ admits a left and a right dual. The choice of left and right duals for each object of a rigid $\mathscr{C}$ defines a left dual functor ${ }^{\vee}$ ? : $\mathscr{C}^{\mathrm{op}} \rightarrow \mathscr{C}$ and a right dual functor $?^{\vee}: \mathscr{C}^{\mathrm{op}} \rightarrow \mathscr{C}$, where $\mathscr{C}^{\text {op }}$ is the opposite category to $\mathscr{C}$ with opposite monoidal structure. The left and right dual functors are strong monoidal. Note that the actual choice of left and right duals is innocuous in the sense that different choices of left (respectively, right) duals define canonically monoidally isomorphic left (respectively, right) dual functors.

There are canonical natural monoidal isomorphisms ${ }^{\vee}\left(X^{\vee}\right) \simeq X \simeq\left({ }^{\vee} X\right)^{\vee}$, but in general the left and right dual functors are not monoidally isomorphic. 
1B. Pivotal categories. A rigid category $\mathscr{C}$ is pivotal (or sovereign) if it is endowed with a monoidal isomorphism between the left and the right dual functors. We may assume that this isomorphism is the identity without loss of generality. In other words, for each object $X$ of $\mathscr{C}$, we have a dual object $X^{*}$ and four morphisms

$$
\begin{aligned}
& \mathrm{ev}_{X}: X^{*} \otimes X \rightarrow \mathbb{1}, \quad \operatorname{coev}_{X}: \mathbb{1} \rightarrow X \otimes X^{*}, \\
& \widetilde{\mathrm{ev}}_{X}: X \otimes X^{*} \rightarrow \mathbb{1}, \quad \widetilde{\operatorname{cov}}_{X}: \mathbb{1} \rightarrow X^{*} \otimes X,
\end{aligned}
$$

such that $\left(X^{*}, \mathrm{ev}_{X}, \operatorname{coev}_{X}\right)$ is a left dual for $X,\left(X^{*}, \widetilde{\mathrm{ev}}_{X}, \widetilde{\operatorname{cov}}_{X}\right)$ is a right dual for $X$, and the induced left and right dual functors coincide as monoidal functors. In particular, the dual $f^{*}: Y^{*} \rightarrow X^{*}$ of any morphism $f: X \rightarrow Y$ in $\mathscr{C}$ is

$$
\begin{aligned}
f^{*} & =\left(\operatorname{ev}_{Y} \otimes \operatorname{id}_{X^{*}}\right)\left(\operatorname{id}_{Y^{*}} \otimes f \otimes \operatorname{id}_{X^{*}}\right)\left(\operatorname{id}_{Y^{*}} \otimes \operatorname{coev}_{X}\right) \\
& =\left(\operatorname{id}_{X^{*}} \otimes \widetilde{e v}_{Y}\right)\left(\operatorname{id}_{X^{*}} \otimes f \otimes \operatorname{id}_{Y^{*}}\right)\left(\widetilde{\operatorname{cov}}_{X} \otimes \operatorname{id}_{Y^{*}}\right) .
\end{aligned}
$$

In what follows, for a pivotal category $\mathscr{b}$, we will suppress the duality constraints $\mathbb{1}^{*} \cong \mathbb{1}$ and $X^{*} \otimes Y^{*} \cong(Y \otimes X)^{*}$. For example, we will write $(f \otimes g)^{*}=g^{*} \otimes f^{*}$ for morphisms $f, g$ in $\mathscr{C}$.

1C. Traces and dimensions. For an endomorphism $f$ of an object $X$ of a pivotal category $\mathscr{C}$, one defines the left and right traces $\operatorname{tr}_{l}(f), \operatorname{tr}_{r}(f) \in \operatorname{End}_{\mathscr{C}}(\mathbb{1})$ by

$$
\operatorname{tr}_{l}(f)=\operatorname{ev}_{X}\left(\operatorname{id}_{X^{*}} \otimes f\right) \widetilde{\operatorname{coev}_{X}} \quad \text { and } \quad \operatorname{tr}_{r}(f)=\widetilde{\operatorname{ev}}_{X}\left(f \otimes \operatorname{id}_{X^{*}}\right) \operatorname{coev}_{X}
$$

They satisfy $\operatorname{tr}_{l}(g h)=\operatorname{tr}_{l}(h g)$ and $\operatorname{tr}_{r}(g h)=\operatorname{tr}_{r}(h g)$ for any morphisms $g: X \rightarrow Y$ and $h: Y \rightarrow X$ in $\mathscr{C}$. Also we have $\operatorname{tr}_{l}(f)=\operatorname{tr}_{r}\left(f^{*}\right)=\operatorname{tr}_{l}\left(f^{* *}\right)$ for any endomorphism $f$ in $\mathscr{C}$. If

$$
\alpha \otimes \operatorname{id}_{X}=\operatorname{id}_{X} \otimes \alpha \quad \text { for all } \alpha \in \operatorname{End}_{\mathscr{C}}(\mathbb{1}) \text { and } X \text { in } \mathscr{b},
$$

then $\operatorname{tr}_{l}, \operatorname{tr}_{r}$ are $\otimes$-multiplicative; that is, $\operatorname{tr}_{l}(f \otimes g)=\operatorname{tr}_{l}(f) \operatorname{tr}_{l}(g)$ and $\operatorname{tr}_{r}(f \otimes g)=$ $\operatorname{tr}_{r}(f) \operatorname{tr}_{r}(g)$ for all endomorphisms $f, g$ in $\mathscr{C}$.

The left and the right dimensions of an object $X$ of $\mathscr{C}$ are defined by $\operatorname{dim}_{l}(X)=$ $\operatorname{tr}_{l}\left(\operatorname{id}_{X}\right)$ and $\operatorname{dim}_{r}(X)=\operatorname{tr}_{r}\left(\operatorname{id}_{X}\right)$. Isomorphic objects have the same dimensions, $\operatorname{dim}_{l}(X)=\operatorname{dim}_{r}\left(X^{*}\right)=\operatorname{dim}_{l}\left(X^{* *}\right)$, and $\operatorname{dim}_{l}(\mathbb{1})=\operatorname{dim}_{r}(\mathbb{1})=\operatorname{id} \mathbb{\mathbb { 1 }}$. If $\mathscr{C}$ satisfies $(1)$, then left and right dimensions are $\otimes$-multiplicative: $\operatorname{dim}_{l}(X \otimes Y)=\operatorname{dim}_{l}(X) \operatorname{dim}_{l}(Y)$ and $\operatorname{dim}_{r}(X \otimes Y)=\operatorname{dim}_{r}(X) \operatorname{dim}_{r}(Y)$ for any $X, Y$ in $\mathscr{C}$.

1D. Penrose graphical calculus. We represent morphisms in a category $\mathscr{C}$ by plane diagrams to be read from the bottom to the top. In a pivotal category $\mathscr{C}$, the diagrams are made of oriented arcs colored by objects of $\mathscr{C}$ and of boxes colored by morphisms of $\mathscr{C}$. The arcs connect the boxes and have no mutual intersections or self-intersections. The identity $\operatorname{id}_{X}$ of an object $X$ of $\mathscr{C}$, a morphism $f: X \rightarrow Y$, 
and the composition of two morphisms $f: X \rightarrow Y$ and $g: Y \rightarrow Z$ are represented respectively as

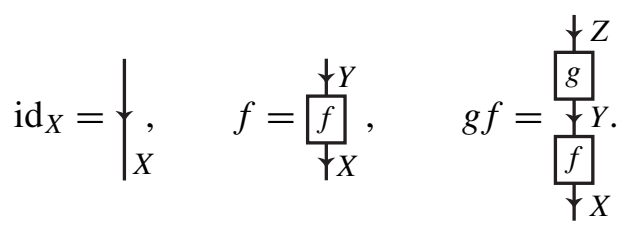

The monoidal product of two morphisms $f: X \rightarrow Y$ and $g: U \rightarrow V$ is represented by juxtaposition:

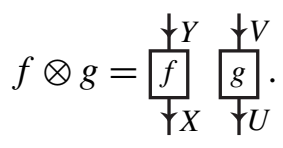

If an arc colored by $X$ is oriented upwards, then the corresponding object in the source/target of morphisms is $X^{*}$. For example, $\operatorname{id}_{X^{*}}$ and a morphism $f: X^{*} \otimes Y \rightarrow$ $U \otimes V^{*} \otimes W$ may be depicted as

$$
\operatorname{id}_{X^{*}}=\uparrow_{X}=\downarrow_{X^{*}} \text { and } f=\frac{\downarrow U \uparrow V \downarrow W}{\uparrow \downarrow_{X}^{W}} .
$$

The duality morphisms are depicted as follows:

$$
\mathrm{ev}_{X}=\bigcap_{x}, \quad \operatorname{coev}_{X}=\bigcup_{x}, \quad \widetilde{\mathrm{ev}}_{X}=\bigcap_{x}, \quad \widetilde{\operatorname{cov}}_{X}=\bigcup_{x} .
$$

The dual of a morphism $f: X \rightarrow Y$ and the traces of a morphism $g: X \rightarrow X$ can be depicted as follows:

$$
f^{*}=\overbrace{Y} f^{X}={ }^{X} f\}_{Y} \text { and } \operatorname{tr}_{l}(g)=X\left\{g, \quad \operatorname{tr}_{r}(g)=g\right\}^{X} .
$$

In a pivotal category, the morphisms represented by the diagrams are invariant under isotopies of the diagrams in the plane keeping fixed the bottom and top endpoints.

1E. Spherical categories. A spherical category is a pivotal category whose left and right traces are equal; i.e., $\operatorname{tr}_{l}(g)=\operatorname{tr}_{r}(g)$ for every endomorphism $g$ of an object. Then $\operatorname{tr}_{l}(g)$ and $\operatorname{tr}_{r}(g)$ are denoted $\operatorname{tr}(g)$ and called the trace of $g$. Similarly, the left and right dimensions of an object $X$ are denoted $\operatorname{dim}(X)$ and called the dimension of $X$.

Note that sphericity can be interpreted in graphical terms: it means that the morphisms represented by closed diagrams are invariant under isotopies of diagrams in the 2-sphere $S^{2}=\mathbb{R}^{2} \cup\{\infty\}$, i.e., are preserved under isotopies pushing arcs of the diagrams across $\infty$. 
1F. Additive categories. Let $\mathbb{k}$ be a commutative ring. A $\mathbb{k}$-additive category is a category where Hom-sets are $\mathbb{k}$-modules, the composition of morphisms is $\mathbb{k}$-bilinear, and any finite family of objects has a direct sum. In particular, such a category has a zero object.

An object $X$ of a $\mathbb{k}$-additive category $\mathscr{C}$ is scalar if the map $\mathbb{k} \rightarrow \operatorname{End}_{\mathscr{C}}(X)$, $\alpha \mapsto \alpha \operatorname{id}_{X}$ is bijective.

A $\mathbb{k}$-additive monoidal category is a monoidal category which is $\mathbb{k}$-additive in such a way that the monoidal product is $\mathbb{k}$-bilinear. Note that a $\mathbb{k}$-additive monoidal category whose unit object $\mathbb{1}$ is scalar satisfies (1) and so its traces $\operatorname{tr}_{l}, \operatorname{tr}_{r}$ are $\mathbb{k}$-linear and $\otimes$-multiplicative.

1G. Fusion categories. A fusion category over a commutative ring $\mathbb{k}$ is a $\mathbb{k}$-additive rigid category $\mathscr{C}$ such that

(a) each object of $\mathscr{b}$ is a finite direct sum of scalar objects;

(b) for any nonisomorphic scalar objects $i, j$ of $\mathscr{C}$, we have $\operatorname{Hom}_{\mathscr{C}}(i, j)=0$;

(c) the set of isomorphism classes of scalar objects of $\mathscr{C}$ is finite;

(d) the unit object $\mathbb{1}$ is scalar.

Let $\mathscr{C}$ be a fusion category. The Hom spaces in $\mathscr{C}$ are free $\mathbb{k}$-modules of finite rank. We identify $\operatorname{End}_{\mathscr{C}}(\mathbb{1})$ with $\mathbb{k}$ via the canonical isomorphism. Given a scalar object $i$ of $\mathscr{C}$, the $i$-isotypical component $X^{(i)}$ of an object $X$ is the largest direct factor of $X$ isomorphic to a direct sum of copies of $i$. The actual number of copies of $i$ is

$$
v_{i}(X)=\operatorname{rank}_{\mathbb{k}} \operatorname{Hom}_{\mathscr{C}}(i, X)=\operatorname{rank}_{\mathbb{k}} \operatorname{Hom}_{\mathscr{C}}(X, i) .
$$

An $i$-decomposition of $X$ is an explicit direct sum decomposition of $X^{(i)}$ into copies of $i$, that is, a family $\left(p_{\alpha}: X \rightarrow i, q_{\alpha}: i \rightarrow X\right)_{\alpha \in A}$ of pairs of morphisms in $\mathscr{C}$ such that

(a) $p_{\alpha} q_{\beta}=\delta_{\alpha, \beta}$ id $_{i}$ for all $\alpha, \beta \in A$,

(b) the set $A$ has $v_{i}(X)$ elements,

where $\delta_{\alpha, \beta}$ is the Kronecker symbol.

A representative set of scalar objects of $\mathscr{C}$ is a set $I$ of scalar objects such that $\mathbb{1} \in I$ and every scalar object of $\mathscr{C}$ is isomorphic to exactly one element of $I$.

Note that if $\mathbb{k}$ is a field, a fusion category over $\mathbb{k}$ is abelian and semisimple. Recall that an abelian category is semisimple if its objects are direct sums of simple ${ }^{1}$ objects.

A pivotal fusion category is spherical (see Section 1E) if and only if the left and right dimension of any of its scalar objects coincide.

${ }^{1} \mathrm{An}$ object of an abelian category is simple if it is nonzero and has no other subobject than the zero object and itself. 
1H. Graphical calculus in pivotal fusion categories. Let $\mathscr{C}$ be a pivotal fusion category. Let $X$ be an object of $\mathscr{C}$ and $i$ be a scalar object of $\mathscr{C}$. Then the tensor

$$
\sum_{\alpha \in A} p_{\alpha} \otimes_{\mathbb{k}} q_{\alpha} \in \operatorname{Hom}_{\mathscr{C}}(X, i) \otimes_{\mathbb{k}} \operatorname{Hom}_{\mathscr{C}}(i, X),
$$

where $\left(p_{\alpha}, q_{\alpha}\right)_{\alpha \in A}$ is an $i$-decomposition of $X$, does not depend on the choice of the $i$-decomposition $\left(p_{\alpha}, q_{\alpha}\right)_{\alpha \in A}$ of $X$. Consequently, a sum of the type

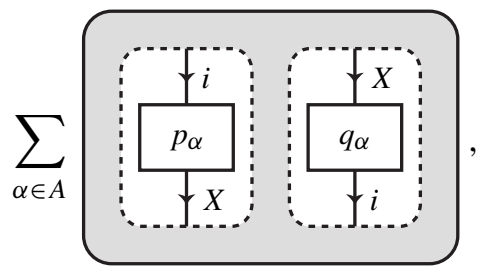

where $\left(p_{\alpha}, q_{\alpha}\right)_{\alpha \in A}$ is an $i$-decomposition of an object $X$ and the gray area does not involve $\alpha$, represents a morphism in $\mathscr{b}$ which is independent of the choice of the $i$-decomposition. We depict it as

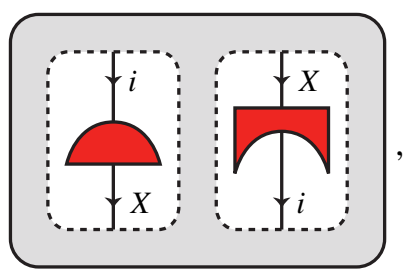

where the two curvilinear boxes should be shaded with the same color. If several such pairs of boxes appear in a picture, they must have different colors. We will also depict
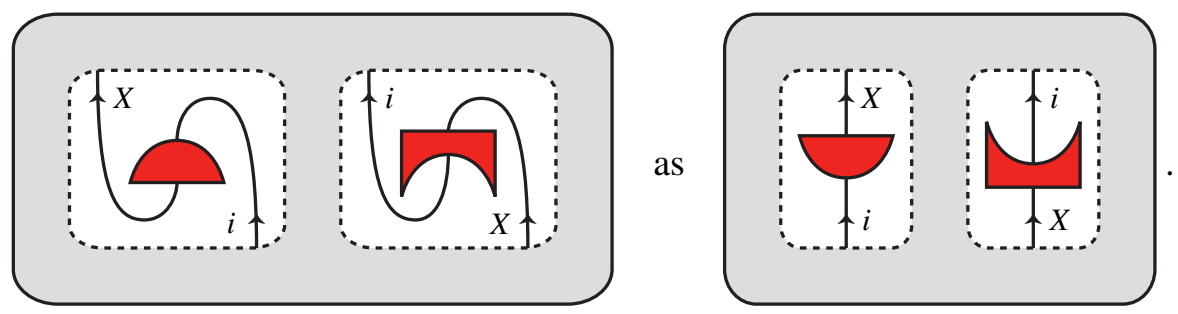

As usual, the edges labeled with $i=\mathbb{1}$ may be erased and then (2) becomes

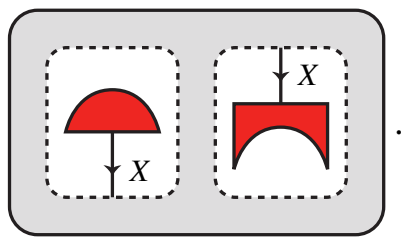


Note also that tensor products of objects may be depicted as bunches of strands. For example,

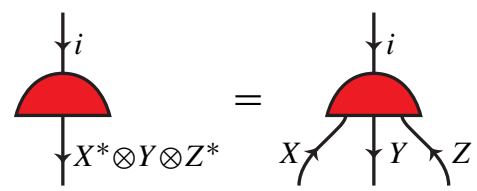

and

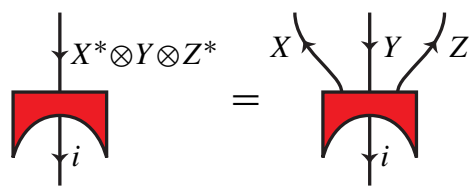

where the equality sign means that the pictures represent the same morphism of $\mathscr{C}$.

1I. Braided and ribbon categories. A braiding in a monoidal category $\mathscr{B}$ is a natural isomorphism $\tau=\left\{\tau_{X, Y}: X \otimes Y \rightarrow Y \otimes X\right\}_{X, Y \in \mathscr{P}}$ such that

$$
\tau_{X, Y \otimes Z}=\left(\operatorname{id}_{Y} \otimes \tau_{X, Z}\right)\left(\tau_{X, Y} \otimes \mathrm{id}_{Z}\right) \quad \text { and } \quad \tau_{X \otimes Y, Z}=\left(\tau_{X, Z} \otimes \mathrm{id}_{Y}\right)\left(\mathrm{id}_{X} \otimes \tau_{Y, Z}\right)
$$

for all $X, Y, Z$ objects of $\mathscr{C}$. These conditions imply that $\tau_{X, \mathbb{1}}=\tau_{\mathbb{1}, X}=\mathrm{id}_{X}$.

A monoidal category endowed with a braiding is said to be braided. The braiding and its inverse are depicted as

$$
\tau_{X, Y}=\searrow_{X Y Y Y} \text { and } \tau_{Y, X}^{-1}={ }_{X y}^{\prime} .
$$

Note that any braided category satisfies the condition (1) of Section 1C.

For any object $X$ of a braided pivotal category $\mathscr{B}$, the morphism

$$
\theta_{X}={ }_{X}{ }^{\prime} \rho=\left(\operatorname{id}_{X} \otimes \tilde{e v}_{X}\right)\left(\tau_{X, X} \otimes \operatorname{id}_{X^{*}}\right)\left(\operatorname{id}_{X} \otimes \operatorname{coev}_{X}\right): X \rightarrow X
$$

is called the twist. The twist is natural in $X$ and invertible, with inverse

$$
\theta_{X}^{-1}=q_{X}^{\prime}=\left(\mathrm{ev}_{X} \otimes \operatorname{id}_{X}\right)\left(\operatorname{id}_{X^{*}} \otimes \tau_{X, X}^{-1}\right)\left(\widetilde{\operatorname{cov}}_{X} \otimes \operatorname{id}_{X}\right): X \rightarrow X .
$$

It satisfies $\theta_{X \otimes Y}=\left(\theta_{X} \otimes \theta_{Y}\right) \tau_{Y, X} \tau_{X, Y}$ for all objects $X, Y$ of $\mathscr{B}$ and $\theta_{\mathbb{1}}=\mathrm{id}_{\mathbb{1}}$.

A ribbon category is a braided pivotal category $\mathscr{B}$ whose twist $\theta$ is self-dual; i.e., $\left(\theta_{X}\right)^{*}=\theta_{X^{*}}$ for any object $X$ of $\mathscr{B}$. This is equivalent to the equality

$$
{ }_{x}^{\prime} \rho=\delta_{y X} .
$$

A ribbon category is spherical.

1J. The center of a monoidal category. Let $\mathscr{C}$ be a monoidal category. A half braiding of $\mathscr{C}$ is a pair $(A, \sigma)$, where $A$ is an object of $\mathscr{C}$ and

$$
\sigma=\left\{\sigma_{X}: A \otimes X \rightarrow X \otimes A\right\}_{X \in \mathscr{C}}
$$

is a natural isomorphism such that

$$
\sigma_{X \otimes Y}=\left(\operatorname{id}_{X} \otimes \sigma_{Y}\right)\left(\sigma_{X} \otimes \mathrm{id}_{Y}\right)
$$


for all $X, Y$ objects of $\mathscr{C}$. This implies that $\sigma_{\mathbb{1}}=\mathrm{id}_{A}$.

The center of $\mathscr{C}$ is the braided category $\mathscr{L}(\mathscr{C})$ defined as follows. The objects of $\mathscr{L}(\mathscr{C})$ are half braidings of $\mathscr{C}$. A morphism $(A, \sigma) \rightarrow\left(A^{\prime}, \sigma^{\prime}\right)$ in $\mathscr{L}(\mathscr{C})$ is a morphism $f: A \rightarrow A^{\prime}$ in $\mathscr{C}$ such that $\left(\operatorname{id}_{X} \otimes f\right) \sigma_{X}=\sigma_{X}^{\prime}\left(f \otimes \operatorname{id}_{X}\right)$ for any object $X$ of $\mathscr{C}$. The unit object of $\mathscr{E}(\mathscr{C})$ is $\mathbb{1}_{\mathscr{L}(\mathscr{C})}=\left(\mathbb{1},\left\{\operatorname{id}_{X}\right\}_{X \in \mathscr{C}}\right)$ and the monoidal product is

$$
(A, \sigma) \otimes(B, \rho)=\left(A \otimes B,\left(\sigma \otimes \mathrm{id}_{B}\right)\left(\mathrm{id}_{A} \otimes \rho\right)\right) .
$$

The braiding $\tau$ in $\mathscr{L}(\mathscr{C})$ is defined by

$$
\tau_{(A, \sigma),(B, \rho)}=\sigma_{B}:(A, \sigma) \otimes(B, \rho) \rightarrow(B, \rho) \otimes(A, \sigma) .
$$

There is a forgetful functor $\mathscr{U}: \mathscr{L}(\mathscr{C}) \rightarrow \mathscr{C}$ assigning to every half braiding $(A, \sigma)$ the underlying object $A$ and acting in the obvious way on the morphisms. This is a strict monoidal functor.

If $\mathscr{C}$ satisfies (1), then $\operatorname{End}_{\mathscr{L}(\mathscr{C})}\left(\mathbb{1}_{\mathscr{L}(\mathscr{C})}\right)=\operatorname{End}_{\mathscr{C}}(\mathbb{1})$.

If $\mathscr{C}$ is rigid, then so is $\mathscr{L}(\mathscr{C})$. If $\mathscr{C}$ is pivotal, then so is $\mathscr{L}(\mathscr{C})$ with $(A, \sigma)^{*}=$ $\left(A^{*}, \sigma^{\natural}\right)$, where

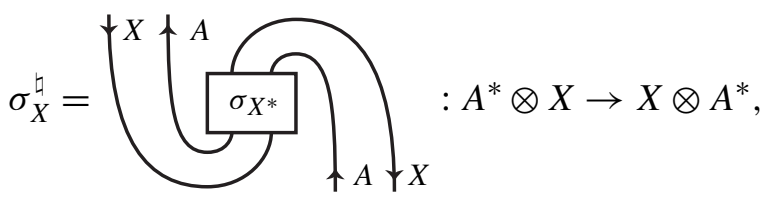

and $\mathrm{ev}_{(A, \sigma)}=\mathrm{ev}_{A}, \operatorname{coev}_{(A, \sigma)}=\operatorname{coev}_{A}, \widetilde{\mathrm{ev}}_{(A, \sigma)}=\widetilde{\mathrm{ev}}_{A}, \widetilde{\operatorname{covv}}_{(A, \sigma)}=\widetilde{\operatorname{cov}}_{A}$. In that case the forgetful functor $U$ preserves (left and right) traces of morphisms and dimensions of objects.

If $\mathscr{C}$ is a $\mathbb{k}$-additive monoidal category, then so is $\mathscr{L}(\mathscr{C})$ and the forgetful functor is $\mathbb{k}$-linear. If $\mathscr{C}$ is an abelian rigid category, then so is $\mathscr{L}(\mathscr{C})$, and the forgetful functor is exact.

If $\mathscr{C}$ is a fusion category over the ring $\mathbb{k}$, then $\mathscr{L}(\mathscr{C})$ is braided $\mathbb{k}$-additive rigid category whose monoidal unit is scalar. If in addition $\mathbb{k}$ is field, then $\mathscr{C}$ is abelian, and so is $\mathscr{L}(\mathscr{C})$.

\section{Main results}

In this section, we state our main results concerning the center of a pivotal fusion category. They are proved in Section 4 . Let $\mathscr{b}$ be a pivotal fusion category over a commutative ring $\mathbb{k}$ and $I$ be a representative set of scalar objects of $\mathscr{C}$. Recall from Section $1 \mathrm{~J}$ that the center $\mathscr{L}(\mathscr{C})$ of $\mathscr{C}$ is a braided $\mathbb{k}$-additive pivotal category whose monoidal unit is scalar.

The coend of a rigid braided category is, if it exists, a Hopf algebra in the category which coacts universally on the objects (see Section $3 \mathrm{C}$ for details). The center 
$\mathscr{L}(\mathscr{C})$ of $\mathscr{C}$ has a coend $(C, \sigma)$, where

$$
C=\bigoplus_{i, j \in I} i^{*} \otimes j^{*} \otimes i \otimes j
$$

and the half braiding $\sigma=\left\{\sigma_{Y}\right\}_{Y \in \mathscr{C}}$ is given by

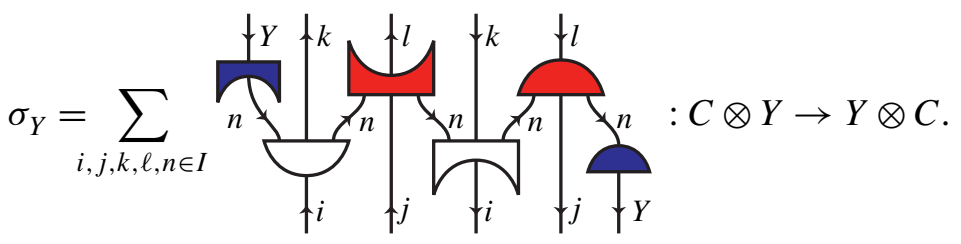

The universal coaction $\delta=\left\{\delta_{M, \gamma}\right\}_{(M, \gamma) \in \mathscr{L}(\mathscr{C})}$ of the coend $(C, \sigma)$ is

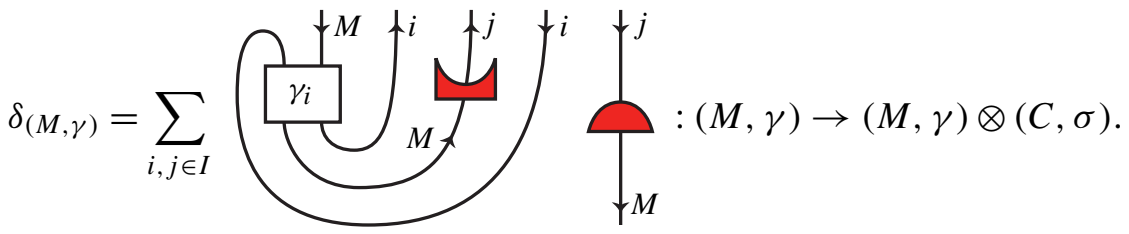

The structural morphisms and the canonical pairing of the Hopf algebra $(C, \sigma)$ can be depicted as follows:

(a) The coproduct $\Delta: C \rightarrow C \otimes C$ :

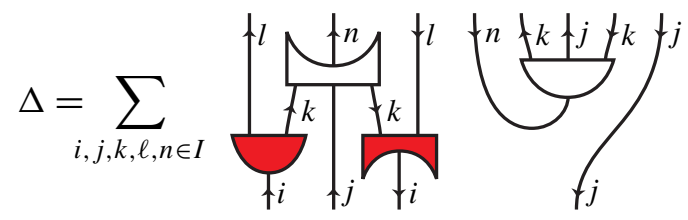

(b) The product $m: C \otimes C \rightarrow C$ :

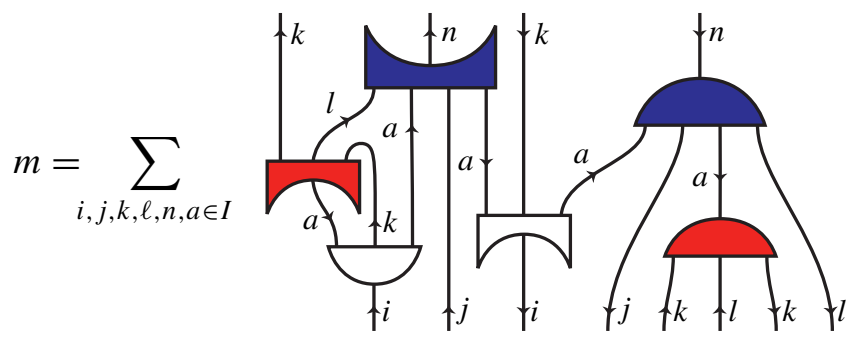

(c) The counit $\varepsilon: C \rightarrow \mathbb{1}: \quad \varepsilon=\sum_{j \in I}: \overbrace{}^{j}$

(d) The unit $u: \mathbb{1} \rightarrow C: \quad u=\sum_{i \in I} \bigodot_{i}:$ 
(e) The antipode $S: C \rightarrow C$ :

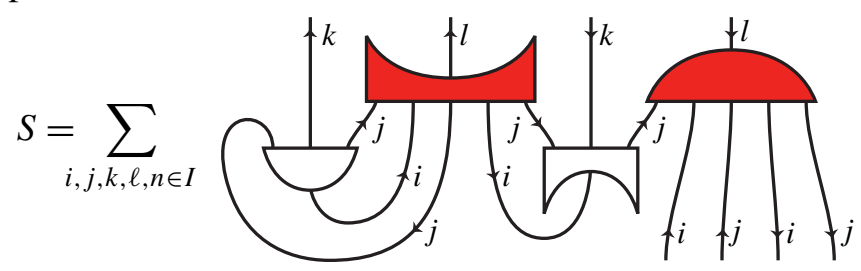

(f) The canonical pairing $\omega: C \otimes C \rightarrow \mathbb{1}$ :

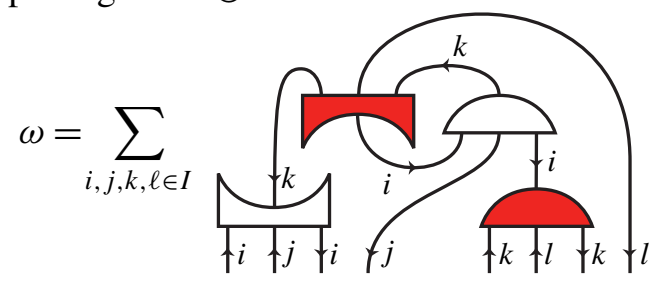

In the pictures, the dotted lines represent $\mathrm{id}_{\mathbb{1}}$ and serve to indicate which direct factor of $C$ is concerned. Moreover,

$$
\Lambda=\sum_{j \in I} \operatorname{dim}_{r}(j): \bigcup_{j}:(\mathbb{1}, \mathrm{id}) \rightarrow(C, \sigma)
$$

is an integral of the Hopf algebra $(C, \sigma)$, which is invariant under the antipode.

By a modular category we mean a braided pivotal category admitting a coend, and whose canonical pairing is nondegenerate (see Section $3 \mathrm{E}$ for details). The dimension of such a category is the dimension of its coend (see Section 3D).

Theorem 2.1. The center $\mathscr{L}(\mathscr{C})$ of $\mathscr{C}$ is modular and has dimension $\operatorname{dim}(\mathscr{C})^{2}$.

The forgetful functor $\mathscr{U}: \mathscr{L}(\mathscr{C}) \rightarrow \mathscr{C}$ has a left adjoint $\mathscr{F}: \mathscr{C} \rightarrow \mathscr{L}(\mathscr{C})$. For an object $X$ of $\mathscr{b}$,

$$
\begin{gathered}
\mathscr{F}(X)=\left(Z(X), \varsigma_{X}=\left\{\varsigma_{X, Y}\right\}_{Y \in \mathscr{C}}\right) \text { where } Z(X)=\bigoplus_{X, Y} i^{*} \otimes X \otimes i \text { and } \\
\left.\Sigma_{i, j \in I} \bigcup_{\uparrow_{i}}^{Y}\right\}_{i} \underbrace{j}_{i}: Z(X) \otimes Y \rightarrow Y \otimes Z(X) .
\end{gathered}
$$

For a morphism $f$ in $\mathscr{C}$,

$$
\mathscr{F}(f)=\sum_{i \in I} \mathrm{id}_{i^{*}} \otimes f \otimes \mathrm{id}_{i} .
$$

By a free half braiding we mean a half braiding of the form $\mathscr{F}(X)$ for some object $X$ of $\mathscr{b}$.

Theorem 2.2. The dimension of $\mathscr{b}$ is invertible in $\mathbb{k}$ if and only if every half braiding is a retract of a free half braiding. 
From Section $1 \mathrm{~J}$, if $\mathbb{k}$ is a field, then $\mathscr{E}(\mathscr{C})$ is abelian.

Corollary 2.3. Assume $k$ is a field.

(a) The center $\mathscr{L}(\mathscr{C})$ is semisimple (as an abelian category) if and only if $\operatorname{dim}(\mathscr{C}) \neq 0$.

(b) Assume $\mathbb{k}$ is algebraically closed. Then $\mathscr{L}(\mathscr{C})$ is a fusion category if and only if $\operatorname{dim}(\mathscr{C}) \neq 0$.

Since the center of a spherical fusion category is ribbon (see, for example, [Turaev and Virelizier 2010, Lemma 10.1]), we recover Müger's theorem:

Corollary 2.4 [Müger 2003, Theorem 1.2]. If $\mathscr{C}$ is a spherical fusion category over an algebraically closed field and $\operatorname{dim}(\mathscr{C}) \neq 0$, then $\mathscr{L}(\mathscr{C})$ is a modular ribbon fusion category (i.e., $\mathscr{E}(\mathscr{C})$ is modular in the sense of [Turaev 1994]).

Note that by [Etingof et al. 2005], the hypothesis $\operatorname{dim}(\mathscr{C}) \neq 0$ of the previous corollary is automatically fulfilled on a field of characteristic zero.

Example 2.5. Let $G$ be a finite group and $\mathbb{k}$ be a commutative ring. The category $\mathscr{C}_{G, \mathbb{k}}$ of $G$-graded free $\mathbb{k}$-modules of finite rank is a spherical fusion category. The dimension of $\mathscr{C}_{G, \mathbb{k}}$ is $\operatorname{dim}\left(\mathscr{C}_{G, \mathbb{k}}\right)=|G| 1_{\mathbb{k}}$, where $|G|$ is the order of $G$. By Theorem 2.1 , the center $\mathscr{L}\left(\mathscr{C}_{G, \mathbb{k}}\right)$ of $\mathscr{C}_{G, \mathbb{k}}$ is modular of dimension $|G|^{2} 1_{\mathbb{k}}$. When $|G|$ is not invertible in $\mathbb{k}$, by Theorem 2.2, there exist half braidings of $\mathscr{C}_{G, \mathbb{k}}$ which are not retracts of any free half braiding. If particular, if $k$ is a field of characteristic $p$ which divides $|G|$, then $\mathscr{L}\left(\mathscr{C}_{G, \mathbb{k}}\right)$ is not semisimple.

\section{Modular categories}

In this section, we clarify some notions used in the previous section. More precisely, in Section 3A, we recall the definition of a Hopf algebra in a braided category and provide a criterion for the nondegeneracy of a Hopf algebra pairing. In Section 3B, we recall the definition of a coend. In Section 3C, we describe the Hopf algebra structure of the coend of a braided rigid category. Sections 3D and 3E are devoted to the definition of respectively the dimension and the modularity of a braided category admitting a coend.

3A. Hopf algebras, pairings, and integrals. Let $\mathscr{B}$ be a braided category, with braiding $\tau$. Recall that a bialgebra in $\mathscr{B}$ is an object $A$ of $\mathscr{B}$ endowed with four morphisms $m: A \otimes A \rightarrow A$ (the product), $u: \mathbb{1} \rightarrow A$ (the unit), $\Delta: A \rightarrow A \otimes A$ (the coproduct), and $\varepsilon: A \rightarrow \mathbb{1}$ (the counit) such that

$$
\begin{gathered}
m\left(m \otimes \mathrm{id}_{A}\right)=m\left(\mathrm{id}_{A} \otimes m\right), \quad m\left(\mathrm{id}_{A} \otimes u\right)=\mathrm{id}_{A}=m\left(u \otimes \mathrm{id}_{A}\right), \\
\left(\Delta \otimes \mathrm{id}_{A}\right) \Delta=\left(\mathrm{id}_{A} \otimes \Delta\right) \Delta, \quad\left(\mathrm{id}_{A} \otimes \varepsilon\right) \Delta=\mathrm{id}_{A}=\left(\varepsilon \otimes \mathrm{id}_{A}\right) \Delta, \\
\Delta m=(m \otimes m)\left(\mathrm{id}_{A} \otimes \tau_{A, A} \otimes \mathrm{id}_{A}\right)(\Delta \otimes \Delta), \\
\Delta u=u \otimes u, \quad \varepsilon m=\varepsilon \otimes \varepsilon, \quad \varepsilon u=\mathrm{id}_{\mathbb{1}} .
\end{gathered}
$$


An antipode for a bialgebra $A$ in $\mathscr{B}$ is a morphism $S: A \rightarrow A$ in $\mathscr{B}$ such that

$$
m\left(S \otimes \operatorname{id}_{A}\right) \Delta=u \varepsilon=m\left(\operatorname{id}_{A} \otimes S\right) \Delta .
$$

If it exists, an antipode is unique. A Hopf algebra in $\mathscr{B}$ is a bialgebra in $\mathscr{B}$ which admits an invertible antipode.

Let $A$ be a Hopf algebra in $\mathscr{B}$. A Hopf pairing for $A$ is a morphism $\omega: A \otimes A \rightarrow \mathbb{1}$ such that

$$
\begin{array}{ll}
\omega\left(m \otimes \mathrm{id}_{A}\right)=\omega\left(\mathrm{id}_{A} \otimes \omega \otimes \mathrm{id}_{A}\right)\left(\mathrm{id}_{A^{\otimes 2}} \otimes \Delta\right), & \omega\left(u \otimes \mathrm{id}_{A}\right)=\varepsilon, \\
\omega\left(\mathrm{id}_{A} \otimes m\right)=\omega\left(\mathrm{id}_{A} \otimes \omega \otimes \mathrm{id}_{A}\right)\left(\Delta \otimes \mathrm{id}_{A^{\otimes 2}}\right), & \omega\left(\mathrm{id}_{A} \otimes u\right)=\varepsilon .
\end{array}
$$

These axioms imply that $\omega\left(S \otimes \mathrm{id}_{A}\right)=\omega\left(\mathrm{id}_{A} \otimes S\right)$.

A Hopf pairing $\omega$ for $A$ is nondegenerate if there exists a morphism $\Omega: \mathbb{1} \rightarrow A \otimes A$ in $\mathscr{B}$ such that

$$
\left(\omega \otimes \mathrm{id}_{A}\right)\left(\mathrm{id}_{A} \otimes \Omega\right)=\mathrm{id}_{A}=\left(\mathrm{id}_{A} \otimes \omega\right)\left(\Omega \otimes \mathrm{id}_{A}\right) .
$$

If such is the case, the morphism $\Omega$ is unique and called the inverse of $\omega$.

A left (respectively, right) integral for $A$ is a morphism $\Lambda: \mathbb{1} \rightarrow A$ such that

$$
m\left(\mathrm{id}_{A} \otimes \Lambda\right)=\Lambda \varepsilon \quad\left(\text { respectively, } m\left(\Lambda \otimes \mathrm{id}_{A}\right)=\Lambda \varepsilon\right) .
$$

A left (respectively, right) cointegral for $A$ is a morphism $\lambda: A \rightarrow \mathbb{1}$ such that

$$
\left(\operatorname{id}_{A} \otimes \lambda\right) \Delta=u \lambda \quad\left(\text { respectively, }\left(\lambda \otimes \mathrm{id}_{A}\right) \Delta=u \lambda\right) .
$$

A (co)integral is two-sided if it is both a left and a right (co)integral.

If $\Lambda$ is a left (respectively, right) integral for $A$, then $S \Lambda$ is a right (respectively, left) integral for $A$. If $\lambda$ is a left (respectively, right) cointegral for $A$, then $\lambda S$ is a right (respectively, left) cointegral for $A$.

Let $\omega$ be a Hopf pairing for $A$ and $\Lambda: \mathbb{1} \rightarrow A$ be a morphism in $\mathscr{B}$. Assume $\omega$ is nondegenerate. Then $\Lambda$ is a left integral for $A$ if and only if $\lambda=\omega\left(\operatorname{id}_{A} \otimes \Lambda\right)$ is a right cointegral for $A$, and $\Lambda$ is a right integral for $A$ if and only if $\lambda=\omega\left(\Lambda \otimes \operatorname{id}_{A}\right)$ is a left cointegral for $A$.

Lemma 3.1. Let $\omega$ be a Hopf pairing for a Hopf algebra A in a braided category $\Re$. Assume there exist morphisms $\Lambda, \Lambda^{\prime}: \mathbb{1} \rightarrow A$ in $\mathscr{B}$ such that

(a) $\omega\left(\Lambda \otimes \mathrm{id}_{A}\right)$ and $\omega\left(\mathrm{id}_{A} \otimes \Lambda^{\prime}\right)$ are left cointegrals for $A$;

(b) $\omega\left(\Lambda \otimes \Lambda^{\prime}\right)$ is invertible in $\operatorname{End}_{\mathscr{B}}(\mathbb{1})$.

Then $\omega$ is nondegenerate, with inverse

$$
\Omega=\omega\left(\Lambda \otimes \Lambda^{\prime}\right)^{-1}\left(S \otimes \mathrm{id}_{A} \otimes \omega\right)\left(\mathrm{id}_{A} \otimes \Delta \Lambda \otimes \mathrm{id}_{A}\right) \Delta \Lambda^{\prime},
$$

and $\Lambda$ and $\Lambda^{\prime}$ are right integrals for $A$. 
Proof. Set $e=\left(S \otimes \mathrm{id}_{A} \otimes \omega\right)\left(\mathrm{id}_{A} \otimes \Delta \Lambda \otimes \mathrm{id}_{A}\right) \Delta \Lambda^{\prime}: \mathbb{1} \rightarrow A \otimes A$. Let us depict the product $m$, coproduct $\Delta$, antipode $S$ of $A$, and the morphisms $\omega, \Lambda, \Lambda^{\prime}$ as follows:

$$
m=h, \quad \Delta=Y, \quad S=\oint, \quad \omega=\bigcap, \quad \Lambda=\text { 只, } \quad \Lambda^{\prime}=\diamond .
$$

Then $\left(\mathrm{id}_{A} \otimes \omega\right)\left(e \otimes \mathrm{id}_{A}\right)=\omega\left(\Lambda \otimes \Lambda^{\prime}\right) \mathrm{id}_{A}$ since

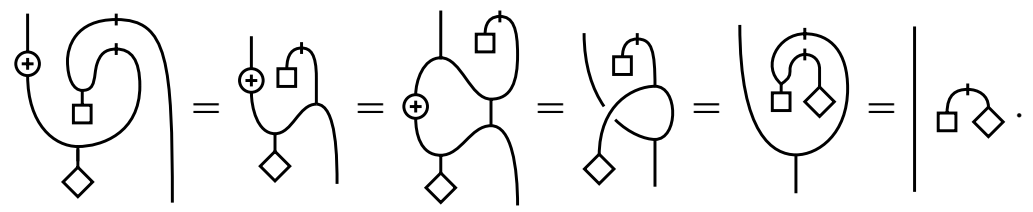

We use the product/coproduct axioms of a Hopf pairing in the first and fourth equalities, the unit axiom and the fact that $\omega\left(\Lambda \otimes \mathrm{id}_{A}\right)$ is a left cointegral in the second equality, the compatibility of $m$ and $\Delta$ and the axiom of the antipode in the third equality, and finally the fact that $\omega\left(\operatorname{id}_{A} \otimes \Lambda^{\prime}\right)$ is a left cointegral and the unit/counit axiom of a Hopf pairing in the last equality. Similarly one shows that $\left(\omega \otimes \mathrm{id}_{A}\right)\left(\operatorname{id}_{A} \otimes e\right)=\omega\left(\Lambda \otimes \Lambda^{\prime}\right) \mathrm{id}_{A}$. Thus $\Omega=\omega\left(\Lambda \otimes \Lambda^{\prime}\right)^{-1} e$ is an inverse of $\omega$.

Finally, since $\omega$ is nondegenerate and $\omega(\Lambda \otimes A)$ and $\omega\left(A \otimes \Lambda^{\prime}\right)$ are left cointegrals, we conclude that $\Lambda$ and $\Lambda^{\prime}$ are right integrals.

3B. Coends. Let $\mathscr{C}$ and $\mathscr{D}$ be categories. A dinatural transformation from a functor $F: \mathscr{D}^{\text {op }} \times \mathscr{D} \rightarrow \mathscr{C}$ to an object $A$ of $\mathscr{C}$ is a family of morphisms in $\mathscr{C}$

$$
d=\left\{d_{Y}: F(Y, Y) \rightarrow A\right\}_{Y \in \mathscr{D}}
$$

such that for every morphism $f: X \rightarrow Y$ in $\mathscr{D}$, we have

$$
d_{X} F\left(f, \operatorname{id}_{X}\right)=d_{Y} F\left(\operatorname{id}_{Y}, f\right): F(Y, X) \rightarrow A .
$$

The composition of such a $d$ with a morphism $\phi: A \rightarrow B$ in $\mathscr{b}$ is the dinatural transformation $\phi \circ d=\left\{\phi \circ d_{X}: F(Y, Y) \rightarrow B\right\}_{Y \in \mathscr{D}}$ from $F$ to $B$. A coend of $F$ is a pair $(C, \rho)$ consisting in an object $C$ of $\mathscr{C}$ and a dinatural transformation $\rho$ from $F$ to $C$ satisfying the following universality condition: every dinatural transformation $d$ from $F$ to an object of $\mathscr{C}$ is the composition of $\rho$ with a morphism in $\mathscr{C}$ uniquely determined by $d$. If $F$ has a coend $(C, \rho)$, then it is unique (up to unique isomorphism). One writes $C=\int^{Y \in \mathscr{D}} F(Y, Y)$. For more on coends, see [Mac Lane 1998].

Remark 3.2. Let $F$ : $\mathscr{D}^{\mathrm{op}} \times \mathscr{D} \rightarrow \mathscr{C}$ be a $\mathbb{k}$-linear functor, where $\mathscr{C}$ is a $\mathbb{k}$-additive category and $\mathscr{D}$ is a fusion category (over $\mathbb{k}$ ). Then $F$ has a coend. More precisely, pick a (finite) representative set $I$ of simple objects of $\mathscr{D}$ and set $C=\bigoplus_{i \in I} F(i, i)$. Let $\rho=\left\{\rho_{Y}: F(Y, Y) \rightarrow C\right\}_{Y \in \mathscr{D}}$ be defined by $\rho_{Y}=\sum_{\alpha} F\left(q_{Y}^{\alpha}, p_{Y}^{\alpha}\right)$, where $\left(p_{Y}^{\alpha}, q_{Y}^{\alpha}\right)_{\alpha}$ is any $I$-partition of $Y$. Then $(C, \rho)$ is a coend of $F$ and each dinatural transformation $d$ from $F$ to any object $A$ of $\mathscr{C}$ is the composition of $\rho$ with $\bigoplus_{i \in I} d_{i}: C \rightarrow A$. 
3C. The coend of a braided rigid category. Let $\mathscr{B}$ be braided rigid category. The coend

$$
C=\int^{Y \in \mathscr{B}}{ }^{\vee} Y \otimes Y
$$

if it exists, is called the coend of $\mathscr{\Re}$.

Assume $\mathscr{B}$ has a coend $C$ and denote by $i_{Y}:{ }^{\vee} Y \otimes Y \rightarrow C$ the corresponding universal dinatural transformation. The universal coaction of $C$ on the objects of $\mathscr{B}$ is the natural transformation $\delta$ defined by

$$
\delta_{Y}=\left(\operatorname{id}_{Y} \otimes i_{Y}\right)\left(\operatorname{coev}_{Y} \otimes \operatorname{id}_{Y}\right): Y \rightarrow Y \otimes C, \quad \text { depicted as } \delta_{Y}=Y_{Y}^{Y} .
$$

According to [Majid 1995], $C$ is a Hopf algebra in $\mathscr{B}$. Its coproduct $\Delta$, product $m$, counit $\varepsilon$, unit $u$, and antipode $S$ with inverse $S^{-1}$ are characterized by the following equalities, where $X, Y \in \mathscr{B}$ :
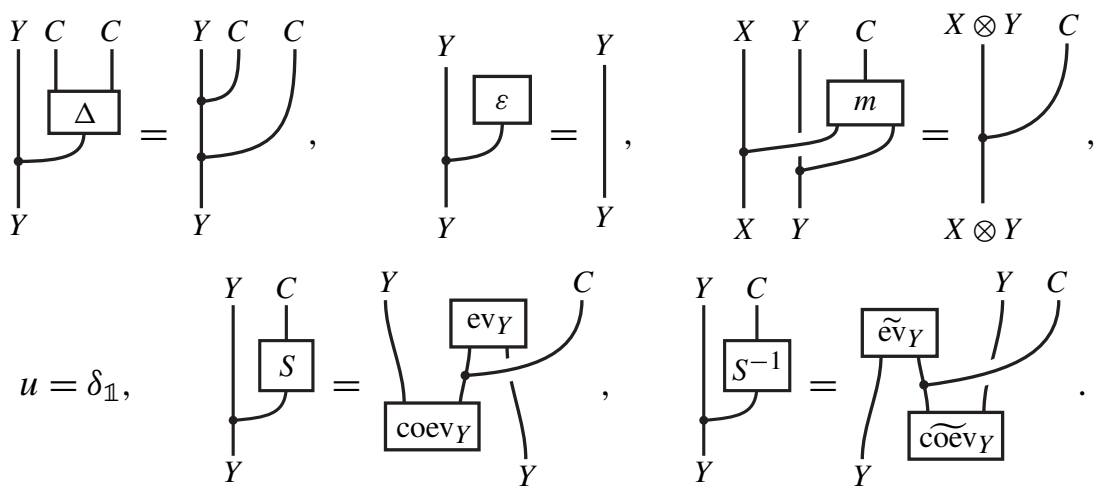

Furthermore, the morphism $\omega: C \otimes C \rightarrow \mathbb{1}$ defined by

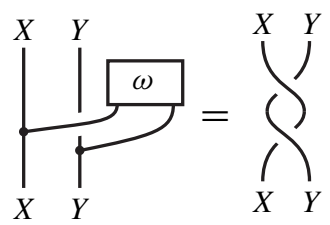

is a Hopf pairing for $C$, called the canonical pairing. Moreover this pairing satisfies the following self-duality condition: $\omega \tau_{C, C}(S \otimes S)=\omega$.

3D. The dimension of a braided pivotal category. Let $\mathscr{B}$ be a braided pivotal category admitting a coend $C$.

Lemma 3.3. The left and right dimensions of $C$ coincide. 
Proof. Let $v=\left\{v_{X}\right\}_{X \in \mathscr{B}}$ be the natural transformation defined by

$$
v_{X}={ }_{x+\rho}^{\downarrow}: X \rightarrow X .
$$

Then $v$ is natural monoidal isomorphism; that is, $v_{X \otimes Y}=v_{X} \otimes v_{Y}$ and $v_{\mathbb{1}}=$ $\mathrm{id}_{\mathbb{1}}$, which implies that $v_{X}{ }^{*}=v_{X^{*}}^{-1}$. The full subcategory $\mathscr{B}_{0}$ of $\mathscr{B}_{3}$ made of the objects $X$ of $\mathscr{B}$ satisfying $\tau_{X}=\mathrm{id}_{X}$ is a ribbon category. Let us prove that the coend $C$ of $\mathscr{B}$ belongs to $\mathscr{B}_{0}$. Denote by $i=\left\{i_{X}: X^{*} \otimes X \rightarrow C\right\}_{X \in \mathscr{B}}$ the universal dinatural transformation associated with $C$. For any object $X$ of $\mathscr{C}$, by naturality and monoidality of $v$ and dinaturality of $i$, the following holds:

$$
v_{C} i_{X}=i_{X} v_{\left(X^{*} \otimes X\right)}=i_{X}\left(v_{X^{*}} \otimes v_{X}\right)=i_{X}\left(v_{X}^{*} v_{X^{*}} \otimes \operatorname{id}_{X}\right)=i_{X} .
$$

So $v_{C}=\operatorname{id}_{C}$; that is, $C$ belongs to $\mathscr{B}_{0}$. Hence the left and right dimensions of $C$ coincide, since $\mathscr{P}_{0}$ is a ribbon category.

We define the dimension of $\mathscr{B}$ as $\operatorname{dim}(\mathscr{B})=\operatorname{dim}_{l}(C)=\operatorname{dim}_{r}(C)$.

This definition agrees with the standard definition of the dimension of a pivotal fusion category. Indeed, any pivotal fusion category $\mathscr{C}$ (over the ring $\mathbb{k}$ ) admits a coend $C=\bigoplus_{i \in I} i^{*} \otimes i$, where $I$ is a (finite) representative set of scalar objects of $\mathscr{C}$, and so

$$
\operatorname{dim}_{l}(C)=\operatorname{dim}_{r}(C)=\sum_{i \in I} \operatorname{dim}_{l}\left(i^{*}\right) \operatorname{dim}_{l}(i)=\sum_{i \in I} \operatorname{dim}_{r}(i) \operatorname{dim}_{l}(i) .
$$

3E. Modular categories. By a modular category, we mean a braided rigid category which admits a coend whose canonical pairing is nondegenerate. Note that when $\mathscr{B}$ is ribbon, this definition coincides with that of a 2-modular category given in [Lyubashenko 1995].

Remark 3.4. Let $\mathscr{B}$ be a braided pivotal fusion category over $\mathbb{k}$. Let $I$ be a representative set of the scalar objects of $\mathscr{B}$. Recall that $C=\bigoplus_{i \in I} i^{*} \otimes i$ is the coend of $\mathscr{B}$. For $i, j \in I$, set

$$
S_{i, j}=\left(\mathrm{ev}_{i} \otimes \widetilde{\mathrm{ev}}_{j}\right)\left(\mathrm{id}_{i} * \tau_{j, i} \tau_{i, j} \otimes \mathrm{id}_{j^{*}}\right)\left(\widetilde{\operatorname{cov}}_{i} \otimes \operatorname{coev}_{j}\right) \in \mathbb{k} .
$$

The matrix $S=\left[S_{i, j}\right]_{i, j \in I}$, called the $S$-matrix of $\mathscr{B}$, is invertible if and only if the canonical pairing of $C$ is nondegenerate. In particular a modular category in the sense of [Turaev 1994] is a ribbon fusion category which is modular in the above sense.

\section{Proofs}

The statements of Section 2 derive directly from the theory of Hopf monads, introduced in [Bruguières and Virelizier 2007] and developed in [Bruguières and 
Virelizier 2012; Bruguières et al. 2011]. Hopf monads generalize Hopf algebras in the setting of general monoidal categories. In Section 4A, we recall some basic definitions concerning Hopf monads. In Section 4B, we give a Hopf monadic description of the center $\mathscr{E}(\mathscr{C})$ of a fusion category $\mathscr{C}$, from which is derived the explicit description of the coend of $\mathscr{L}(\mathscr{C})$. In Section $4 \mathrm{C}$, we prove a "handleslide" property for pivotal fusion categories. In Section $4 \mathrm{D}$, we use the explicit description of the coend of $\mathscr{L}(\mathscr{C})$ to prove Theorem 2.1 and prove that the morphism $\Lambda$ of (6) is an integral invariant under the antipode. Sections $4 \mathrm{E}$ and $4 \mathrm{~F}$ are devoted to the proofs of Theorem 2.2 and Corollary 2.3, respectively.

4A. Hopf monads and their modules. Let $\mathscr{b}$ be a category. A monad on $\mathscr{b}$ is a monoid in the category of endofunctors of $\mathscr{C}$, that is, a triple $(T, \mu, \eta)$ consisting of a functor $T: \mathscr{C} \rightarrow \mathscr{C}$ and two natural transformations

$$
\mu=\left\{\mu_{X}: T^{2}(X) \rightarrow T(X)\right\}_{X \in \mathscr{C}} \quad \text { and } \quad \eta=\left\{\eta_{X}: X \rightarrow T(X)\right\}_{X \in \mathscr{C}},
$$

called the product and the unit of $T$, such that, for any object $X$ of $\mathscr{C}$,

$$
\mu_{X} T\left(\mu_{X}\right)=\mu_{X} \mu_{T(X)} \quad \text { and } \quad \mu_{X} \eta_{T(X)}=\mathrm{id}_{T(X)}=\mu_{X} T\left(\eta_{X}\right) .
$$

Given a monad $T=(T, \mu, \eta)$ on $\mathscr{b}$, a $T$-module in $\mathscr{C}$ is a pair $(M, r)$ where $M$ is an object of $\mathscr{C}$ and $r: T(M) \rightarrow M$ is a morphism in $\mathscr{C}$ such that $r T(r)=r \mu_{M}$ and $r \eta_{M}=\mathrm{id}_{M}$. A morphism from a $T$-module $(M, r)$ to a $T$-module $(N, s)$ is a morphism $f: M \rightarrow N$ in $\mathscr{C}$ such that $f r=s T(f)$. This defines the category $\mathscr{C}^{T}$ of $T$-modules in $\mathscr{b}$ with composition induced by that in $\mathscr{C}$. We define a forgetful functor $U_{T}: \mathscr{C}^{T} \rightarrow \mathscr{C}$ by $U_{T}(M, r)=M$ and $U_{T}(f)=f$. The forgetful functor $U_{T}$ has a left adjoint $F_{T}: \mathscr{C} \rightarrow \mathscr{C}^{T}$, called the free module functor, defined by $F_{T}(X)=$ $\left(T(X), \mu_{X}\right)$ and $F_{T}(f)=T(f)$. Note that if $\mathscr{C}$ is $\mathbb{k}$-additive and $T$ is $\mathbb{k}$-linear (that is, $T$ induces $\mathbb{k}$-linear maps on Hom spaces), then the category $\mathscr{C}^{T}$ is $\mathbb{k}$-additive and the functors $U_{T}$ and $F_{T}$ are $\mathbb{k}$-linear.

Let $\mathscr{C}$ be a monoidal category. A bimonad on $\mathscr{C}$ is a monoid in the category of comonoidal endofunctors of $\mathscr{C}$. In other words, a bimonad on $\mathscr{C}$ is a monad $(T, \mu, \eta)$ on $\mathscr{C}$ such that the functor $T: \mathscr{C} \rightarrow \mathscr{C}$ and the natural transformations $\mu$ and $\eta$ are comonoidal; that is, $T$ comes equipped with a natural transformation $T_{2}=\left\{T_{2}(X, Y): T(X \otimes Y) \rightarrow T(X) \otimes T(Y)\right\}_{X, Y \in \mathscr{C}}$ and a morphism $T_{0}: T(\mathbb{1}) \rightarrow \mathbb{1}$ such that

$$
\begin{gathered}
\left(\operatorname{id}_{T(X)} \otimes T_{2}(Y, Z)\right) T_{2}(X, Y \otimes Z)=\left(T_{2}(X, Y) \otimes \operatorname{id}_{T(Z)}\right) T_{2}(X \otimes Y, Z) ; \\
\left(\operatorname{id}_{T(X)} \otimes T_{0}\right) T_{2}(X, \mathbb{1})=\mathrm{id}_{T(X)}=\left(T_{0} \otimes \mathrm{id}_{T(X)}\right) T_{2}(\mathbb{1}, X) ; \\
T_{2}(X, Y) \mu_{X \otimes Y}=\left(\mu_{X} \otimes \mu_{Y}\right) T_{2}(T(X), T(Y)) T\left(T_{2}(X, Y)\right) ; \\
T_{2}(X, Y) \eta_{X \otimes Y}=\eta_{X} \otimes \eta_{Y} .
\end{gathered}
$$


For any bimonad $T$ on $\mathscr{C}$, the category of $T$-modules $\mathscr{C}^{T}$ has a monoidal structure with unit object $\left(\mathbb{1}, T_{0}\right)$ and with tensor product

$$
(M, r) \otimes(N, s)=\left(M \otimes N,(r \otimes s) T_{2}(M, N)\right) .
$$

Note that the forgetful functor $U_{T}: \mathscr{C}^{T} \rightarrow \mathscr{C}$ is strict monoidal.

Given a bimonad $(T, \mu, \eta)$ on $\mathscr{C}$ and objects $X, Y \in \mathscr{C}$, one defines the left fusion operator

$$
H_{X, Y}^{l}=\left(T(X) \otimes \mu_{Y}\right) T_{2}(X, T(Y)): T(X \otimes T(Y)) \rightarrow T(X) \otimes T(Y)
$$

and the right fusion operator

$$
H_{X, Y}^{r}=\left(\mu_{X} \otimes T(Y)\right) T_{2}(T(X), Y): T(T(X) \otimes Y) \rightarrow T(X) \otimes T(Y) .
$$

A Hopf monad on $\mathscr{C}$ is a bimonad on $\mathscr{C}$ whose left and right fusion operators are isomorphisms for all objects $X, Y$ of $\mathscr{C}$. When $\mathscr{C}$ is a rigid category, a bimonad $T$ on $\mathscr{C}$ is a Hopf monad if and only if the category $\mathscr{C}^{T}$ is rigid. The structure of a rigid category in $\mathscr{C}^{T}$ can then be encoded in terms of natural transformations

$$
s^{l}=\left\{s_{X}^{l}: T\left({ }^{\vee} T(X)\right) \rightarrow{ }^{\vee} X\right\}_{X \in \mathscr{C}} \quad \text { and } \quad s^{r}=\left\{s_{X}^{r}: T\left(T(X)^{\vee}\right) \rightarrow X^{\vee}\right\}_{X \in \mathscr{C}},
$$

called the left and right antipodes. They are computed from the fusion operators:

$$
\begin{aligned}
& s_{X}^{l}=\left(T_{0} T\left(\operatorname{ev}_{T(X)}\right)\left(H_{\vee}^{l} T(X), X\right)^{-1} \otimes{ }^{\vee} \eta_{X}\right)\left(\operatorname{id}_{T\left({ }^{\vee} T(X)\right)} \otimes \operatorname{coev}_{T(X)}\right) ; \\
& s_{X}^{r}=\left(\eta_{X}^{\vee} \otimes T_{0} T\left(\widetilde{\operatorname{ev}}_{T(X)}\right)\left(H_{X, T(X)^{\vee}}^{r}\right)^{-1}\right)\left(\widetilde{\operatorname{cov}}_{T(X)} \otimes \operatorname{id}_{T\left(T(X)^{\vee}\right)}\right) .
\end{aligned}
$$

The left and right duals of any $T$-module $(M, r)$ are then defined by

$$
{ }^{\vee}(M, r)=\left({ }^{\vee} M, s_{M}^{l} T\left({ }^{\vee} r\right)\right) \text { and }(M, r)^{\vee}=\left(M^{\vee}, s_{M}^{r} T\left(r^{\vee}\right)\right) \text {. }
$$

A quasitriangular Hopf monad on $\mathscr{C}$ is a Hopf monad $T$ on $\mathscr{b}$ equipped with an $\mathrm{R}$-matrix, that is, a natural transformation

$$
R=\left\{R_{X, Y}: X \otimes Y \rightarrow T(Y) \otimes T(X)\right\}_{X, Y \in \mathscr{C}}
$$

satisfying appropriate axioms which ensure that the natural transformation $\tau=$ $\left\{\tau_{(M, r),(N, s)}\right\}_{(M, r),(N, s) \in \mathscr{C}^{T}}$ defined by

$$
\tau_{(M, r),(N, s)}=(s \otimes r) R_{M, N}:(M, r) \otimes(N, s) \rightarrow(N, s) \otimes(M, r)
$$

form a braiding in the category $\mathscr{C}^{T}$ of $T$-modules. 
4B. The coend of the center of a fusion category. Let $\mathscr{C}$ be a pivotal fusion category (over the ring $\mathbb{k}$ ), with a representative set of scalar objects $I$. For each object $X$ of $\mathscr{C}$, by Remark 3.2, the $\mathbb{k}$-linear functor $\mathscr{C}^{\text {op }} \times \mathscr{C} \rightarrow \mathscr{C}$, defined by $(U, V) \mapsto U^{*} \otimes X \otimes V$, has a coend

$$
Z(X)=\bigoplus_{i \in I} i^{*} \otimes X \otimes i
$$

with dinatural transformation $\rho_{X}=\left\{\rho_{X, Y}\right\}_{Y \in \mathscr{C}}$ given by

$$
\rho_{X, Y}=\sum_{i \in I} \underbrace{\uparrow_{\dagger}}_{\uparrow_{Y}} \overbrace{X \uparrow_{Y}}^{\downarrow^{i}}: Y^{*} \otimes X \otimes Y \rightarrow Z(X) .
$$

The correspondence $X \mapsto Z(X)$ extends to a functor $Z: \mathscr{C} \rightarrow \mathscr{C}$. By Theorem 6.4 and Section 9.2 of [Bruguières and Virelizier 2012], $Z$ is a quasitriangular Hopf monad on $\mathscr{C}$, with structural morphisms as follows (the dotted lines represent $\mathrm{id}_{\mathbb{1}}$ ):

$$
\begin{aligned}
& Z_{2}(X, Y)=\sum_{i \in I} \underbrace{}_{i} \bigcup_{Y} / L_{i}: Z(X \otimes Y) \rightarrow Z(X) \otimes Z(Y), \\
& Z_{0}=\sum_{i \in I} \bigcap^{i}: Z(\mathbb{1}) \rightarrow \mathbb{1},
\end{aligned}
$$

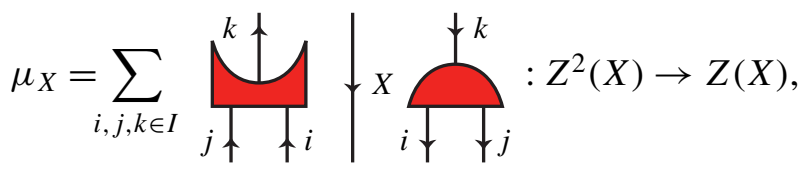

$$
\begin{aligned}
& \eta_{X}=\left.\right|_{\bigvee_{X}} \vdots: X \rightarrow X=\mathbb{1}^{*} \otimes X \otimes \mathbb{1} \hookrightarrow Z(X), \\
& s_{X}^{l}=s_{X}^{r}=\sum_{i, j \in I^{j}} \bigcap_{\uparrow_{i}} \uparrow_{i^{*}}^{x} \widehat{\bigcap}_{\uparrow_{j}}: Z\left(Z(X)^{*}\right) \rightarrow X^{*}, \\
& R_{X, Y}=\sum_{i \in I} \underbrace{\downarrow_{Y}} \int_{\downarrow_{Y}}^{\uparrow_{Y}}: X \otimes Y \rightarrow Z(Y) \otimes Z(X) .
\end{aligned}
$$

In particular, the category $\mathfrak{C}^{Z}$ of $Z$-modules is a braided pivotal category. By [Bruguières and Virelizier 2012, Theorem 6.5], the functor

$$
\Phi:\left\{\begin{aligned}
\mathscr{C}^{Z} & \rightarrow \mathscr{L}(\mathscr{C}) \\
(M, r) & \mapsto(M, \sigma) \\
f & \mapsto f
\end{aligned} \quad \text { where } \quad \sigma_{Y}=\left.\sum_{i \in I}\right|_{f_{Y}} ^{+\downarrow_{i}}\right.
$$


is an isomorphism of braided pivotal categories. Note that this isomorphism is a "fusion" version of the braided isomorphism $\mathscr{L}\left(\bmod _{H}\right) \simeq \bmod _{D(H)}$ between the center of the category of modules over a finite-dimensional Hopf algebra $H$ and the category of modules over the Drinfeld double $D(H)$ of $H$. Now by [Bruguières and Virelizier 2012, Section 6.3], the coend of $\mathscr{b}^{Z}$ is $(C, \alpha)$, where

$$
C=\bigoplus_{i, j \in I} i^{*} \otimes j^{*} \otimes i \otimes j \quad \text { and } \alpha=\sum_{i, j, k, l, n \in I} \overbrace{\uparrow_{n}}
$$

with universal dinatural transformation $\iota=\left\{\iota_{(M, r)}\right\}_{(M, r) \in \mathscr{C}^{Z}}$ given by

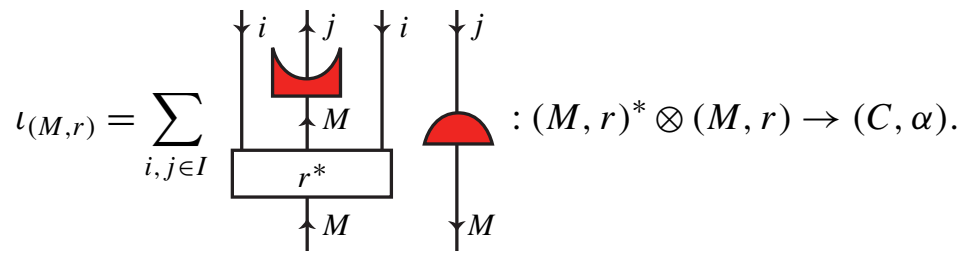

Thus $(C, \sigma)=\Phi(C, \alpha)$ is the coend of $\mathscr{E}(\mathscr{C})$, with universal dinatural transformation $\left\{\Phi\left(\iota_{\Phi^{-1}(M, \gamma)}\right)\right\}_{(M, \gamma) \in \mathscr{L}(\mathscr{C})}$. Using the description of $\Phi$ and the definition of the universal coaction given in (7), we obtain that the half braiding $\sigma$ is given by (4) and that the universal coaction of $(C, \sigma)$ is given by (5). Finally, recall from Section 3C that $(C, \alpha)$ is a Hopf algebra in $\mathscr{C}^{Z}$ endowed with a canonical Hopf algebra pairing. By [Bruguières and Virelizier 2012, Section 9.3], the structural morphisms of $(C, \alpha)$ are those given on pages 10 and 11, items (a)-(f). These structural morphisms are also those of $(C, \sigma)$, since $\Phi$ is the identity on morphisms.

4C. Slope and handleslide in pivotal fusion categories. Let $\mathscr{C}$ be a pivotal fusion category. Recall that the left and right dimensions of a scalar object of $\mathscr{b}$ are invertible. The slope of a scalar object $i$ is the invertible scalar sl $(i)$ defined by

$$
\operatorname{sl}(i)=\frac{\operatorname{dim}_{l}(i)}{\operatorname{dim}_{r}(i)} .
$$

The slope of an object $X$ of $\mathscr{C}$ is the morphism $\mathrm{SL}_{X}: X \rightarrow X$ defined as

$$
\mathrm{SL}_{X}=\sum_{\alpha \in A} \operatorname{sl}\left(i_{\alpha}\right) q_{\alpha} p_{\alpha}
$$

where $\left(p_{\alpha}: X \rightarrow i_{\alpha}, q_{\alpha}: i_{\alpha} \rightarrow X\right)_{\alpha \in A}$ is a decomposition of $X$ as a sum of scalar objects, that is, a family of pairs of morphisms such that $i_{\alpha}$ is scalar for every $\alpha \in A$, $p_{\alpha} q_{\beta}=\delta_{\alpha, \beta} \operatorname{id}_{i_{\alpha}}$ for all $\alpha, \beta \in A$, and $\operatorname{id}_{X}=\sum_{\alpha \in A} q_{\alpha} p_{\alpha}$. The morphism SL $\mathrm{SL}_{X}$ does not depend on the choice of the decomposition of $X$ into scalar objects. Note that 
$\mathrm{SL}_{X}$ is invertible with inverse

$$
\mathrm{SL}_{X}^{-1}=\sum_{\alpha \in A} \operatorname{sl}\left(i_{\alpha}\right)^{-1} q_{\alpha} p_{\alpha} .
$$

The family $\mathrm{SL}=\left\{\mathrm{SL}_{X}: X \rightarrow X\right\}_{X \in \mathscr{C}}$ is a monoidal natural automorphism of the identity functor $1_{\mathscr{C}}$ of $\mathscr{C}$, called the slope operator of $\mathscr{C}$. In particular

$$
\mathrm{SL}_{Y} f=f \mathrm{SL}_{X} \quad \text { and } \quad \mathrm{SL}_{X \otimes Y}=\mathrm{SL}_{X} \otimes \mathrm{SL}_{Y}
$$

for all objects $X, Y$ of $\mathscr{C}$ and all morphism $f: X \rightarrow Y$. The slope operator relates the left and right traces: for any endomorphism $f$ of an object of $\mathscr{C}$,

$$
\operatorname{tr}_{l}(f)=\operatorname{tr}_{r}\left(f \mathrm{SL}_{X}\right) .
$$

Note that $\mathscr{b}$ is spherical if and only its slope operator is the identity.

Lemma 4.1. Let I be a representative set of scalar objects of $\mathscr{C}$.

(a) For any object $X$ of $\mathscr{C}$,

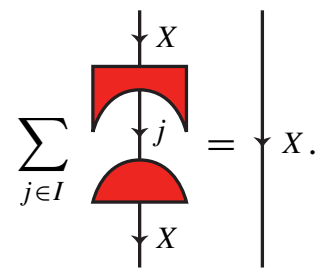

(b) For $i, j \in I$ and $X, Y$ objects of $\mathscr{C}$,

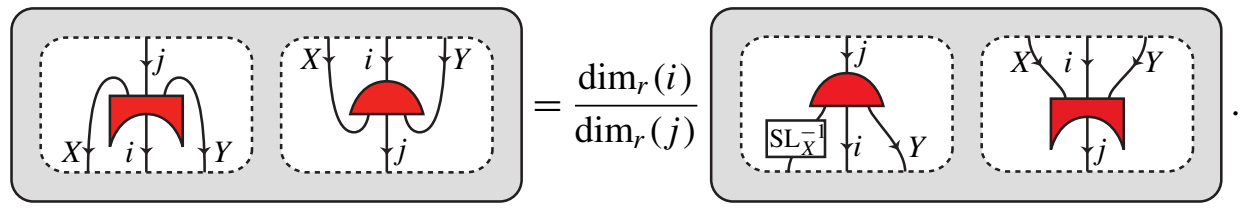

(c) For $i \in I$ and $X, Y$ objects of $\mathscr{C}$,

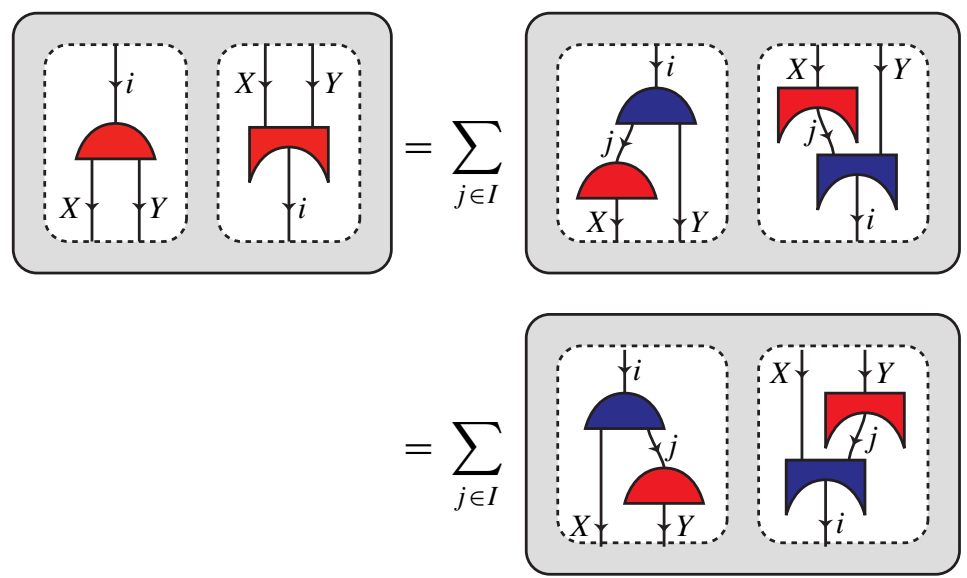

provided there are no $j$-colored strands in the gray area. 
(d) For all $i, j \in I$,

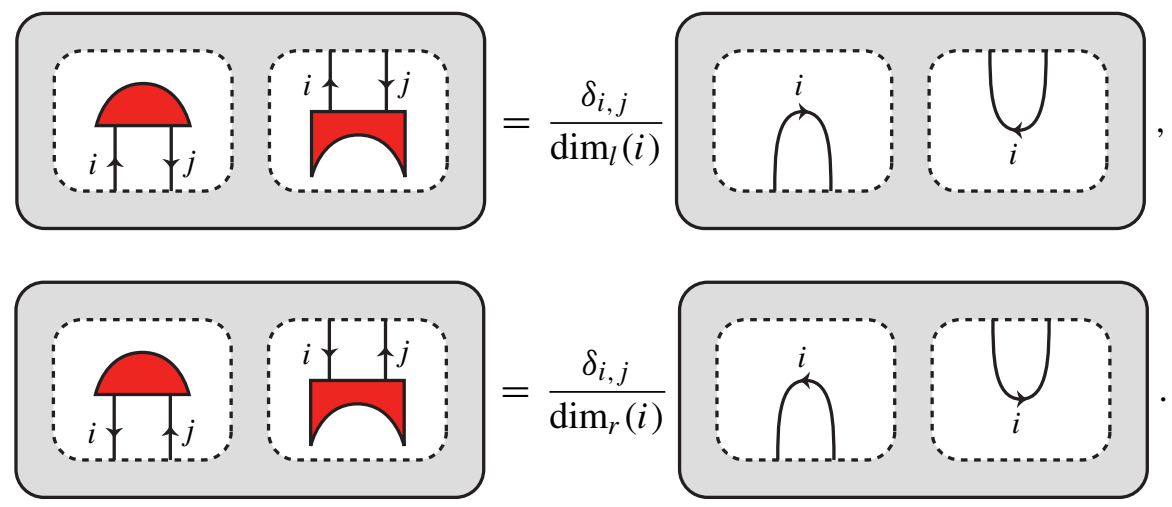

Proof. Part (a) follows directly from the definitions. We prove (b). Let $\left(p_{\alpha}, q_{\alpha}\right)_{\alpha \in A}$ be an $i$-decomposition of $X^{*} \otimes j \otimes Y^{*}$. For $\alpha, \beta \in A$, set

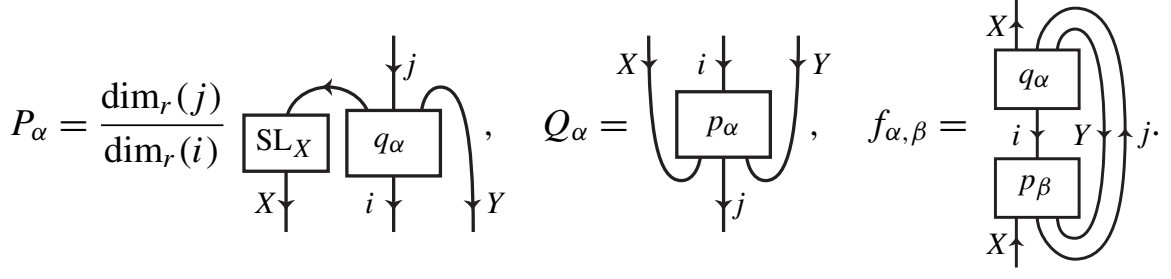

We need to prove that $\left(P_{\alpha}, Q_{\alpha}\right)_{\alpha \in A}$ is a $j$-decomposition of $X \otimes i \otimes Y$. Let $\alpha, \beta \in A$. Since $\left(\mathrm{SL}_{X}\right)^{*}=\mathrm{SL}_{X^{*}}^{-1}$ and using (9), we obtain

$$
\begin{aligned}
P_{\alpha} Q_{\beta} & =\frac{\operatorname{tr}_{r}\left(P_{\alpha} Q_{\beta}\right)}{\operatorname{dim}_{r}(j)} \mathrm{id}_{j}=\frac{\operatorname{tr}_{l}\left(f_{\alpha, \beta} \operatorname{SL}_{X^{*}}^{-1}\right)}{\operatorname{dim}_{r}(i)} \mathrm{id}_{j}=\frac{\operatorname{tr}_{r}\left(f_{\alpha, \beta}\right)}{\operatorname{dim}_{r}(i)} \mathrm{id}_{j} \\
& =\frac{\operatorname{tr}_{r}\left(q_{\alpha} p_{\beta}\right)}{\operatorname{dim}_{r}(i)} \mathrm{id}_{j}=\frac{\operatorname{tr}_{r}\left(p_{\beta} q_{\alpha}\right)}{\operatorname{dim}_{r}(i)} \mathrm{id}_{j}=\frac{\operatorname{tr}_{r}\left(\delta_{\alpha, \beta} \operatorname{id}_{i}\right)}{\operatorname{dim}_{r}(i)} \mathrm{id}_{j}=\delta_{\alpha, \beta} \mathrm{id}_{j} .
\end{aligned}
$$

We conclude using that $\operatorname{card}(A)=v_{i}\left(X^{*} \otimes j \otimes Y^{*}\right)=v_{j}(X \otimes i \otimes Y)$.

Part (c) reflects the canonical isomorphisms

$$
\begin{aligned}
\operatorname{Hom}_{\mathscr{C}}(X \otimes Y, i) & \cong \bigoplus_{j \in I} \operatorname{Hom}_{\mathscr{C}}(X, j) \otimes_{\mathbb{k}} \operatorname{Hom}_{\mathscr{C}}(j \otimes Y, i) \\
& \cong \bigoplus_{j \in I} \operatorname{Hom}_{\mathscr{C}}(X \otimes j, i) \otimes_{\mathbb{k}} \operatorname{Hom}_{\mathscr{C}}(Y, j),
\end{aligned}
$$

and part (d) is a direct consequence of the duality axioms.

4D. Proof of Theorem 2.1 and of the integrality of $\Lambda$. Recall that $\mathscr{L}(\mathscr{C})$ is a braided pivotal category which has a coend $(C, \sigma)$ with $C=\bigoplus_{i, j \in I} i^{*} \otimes j^{*} \otimes i \otimes j$. Therefore its dimension is well-defined and 


$$
\begin{aligned}
\operatorname{dim} \mathscr{L}(\mathscr{C}) & =\operatorname{dim}_{l}(C, \sigma)=\operatorname{dim}_{l}(C)=\operatorname{dim}_{l}\left(\sum_{i, j \in I} i^{*} \otimes j^{*} \otimes i \otimes j\right) \\
& =\sum_{i, j \in I} \operatorname{dim}_{l}\left(i^{*}\right) \operatorname{dim}_{l}\left(j^{*}\right) \operatorname{dim}_{l}(i) \operatorname{dim}_{l}(j) \\
& =\left(\sum_{i \in I} \operatorname{dim}_{r}(i) \operatorname{dim}_{l}(i)\right)\left(\sum_{j \in I} \operatorname{dim}_{r}(j) \operatorname{dim}_{l}(j)\right)=\operatorname{dim}(\mathscr{C})^{2}
\end{aligned}
$$

Let us prove that the canonical pairing of the coend $(C, \sigma)$ is nondegenerate. Define the morphism $\lambda: C \rightarrow \mathbb{1}$ as follows and recall the definition of the morphism $\Lambda: \mathbb{1} \rightarrow C$ of (6):

$$
\lambda=\sum_{i \in I} \operatorname{dim}_{r}(i) \overbrace{(}^{i} \quad \text { and } \Lambda=\sum_{j \in I} \operatorname{dim}_{r}(j) \quad \bigcup_{j} .
$$

Firstly, $\Lambda$ is a morphism in $\mathscr{E}(\mathscr{C})$ from $\mathbb{1}_{\mathscr{E}(\mathscr{C})}=(\mathbb{1}$, id $)$ to $(C, \sigma)$. Indeed, using the description of the half braiding $\sigma$ given in (4), we obtain that for any object $Y$ of $\mathscr{b}$,

$$
\begin{aligned}
\sigma_{Y}\left(\Lambda \otimes \mathrm{id}_{Y}\right) & =\sum_{j, k, \ell, n \in I} \operatorname{dim}_{r}(j) \\
& =\sum_{j, k, \ell, n \in I} \frac{\operatorname{dim}_{r}(\ell)}{\operatorname{sl}(n)} \\
& =\sum_{k, \ell, n \in I} \frac{\operatorname{dim}_{r}(\ell)}{\operatorname{sl}(n)} \\
& =\sum_{\ell, n \in I} \frac{\operatorname{dim}_{r}(\ell)}{\operatorname{sl}_{(n)}} \\
& =\sum_{\ell, n \in I} \operatorname{dim}_{r}(\ell) \\
& =\operatorname{id}_{Y} \otimes \underbrace{}_{n}
\end{aligned}
$$


Secondly, $\lambda$ and $\Lambda$ satisfy $\omega\left(\operatorname{id}_{C} \otimes \Lambda\right)=\lambda=\omega\left(\Lambda \otimes \operatorname{id}_{C}\right)$. Indeed, using the description of the canonical pairing $\omega$ given in item (f) on page 11 , we obtain

$\omega\left(\operatorname{id}_{C} \otimes \Lambda\right)=\sum_{i, j, \ell \in I} \operatorname{dim}_{r}(\ell)$
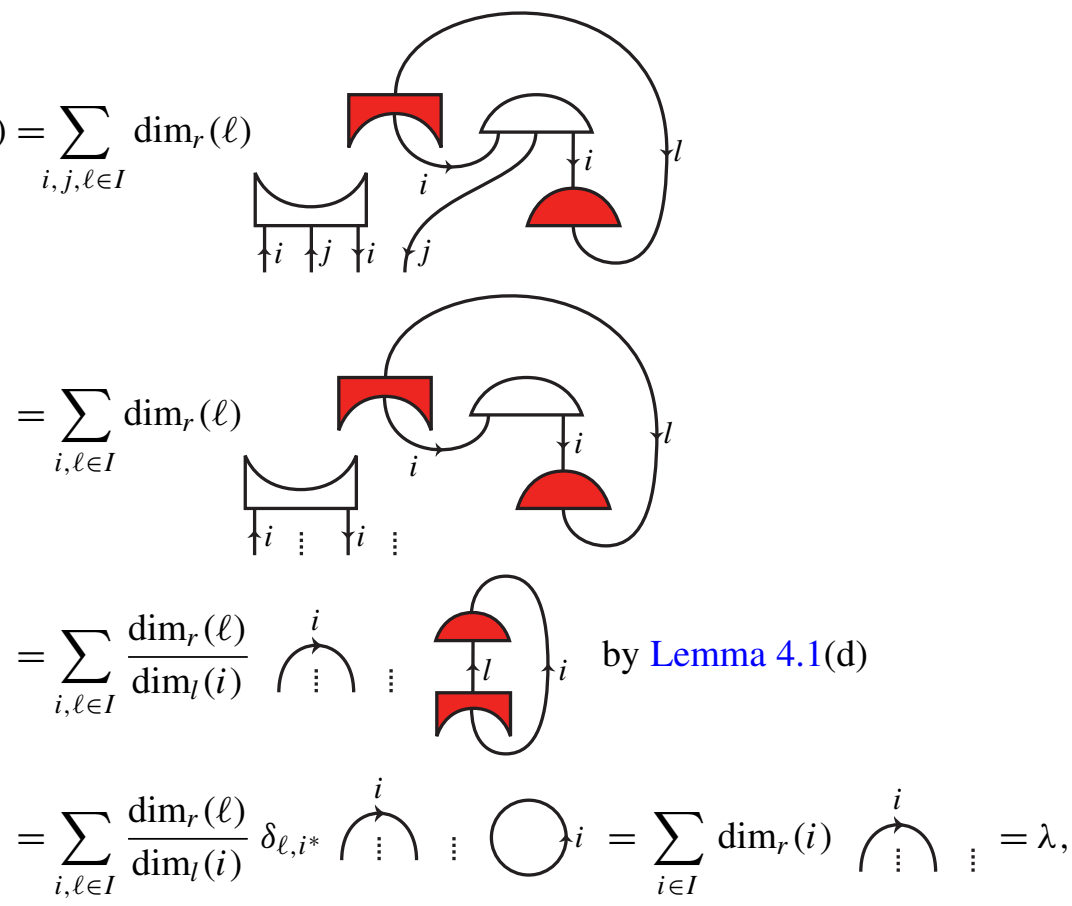

and similarly

$$
\begin{aligned}
& \omega\left(\Lambda \otimes \operatorname{id}_{C}\right)=\sum_{j, k, \ell \in I} \operatorname{dim}_{r}(j)
\end{aligned}
$$

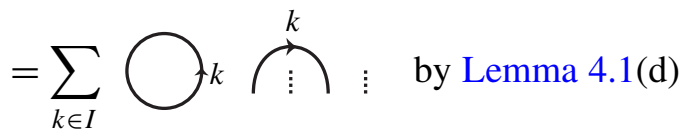

$$
\begin{aligned}
& =\sum_{k \in I} \operatorname{dim}_{r}(k) \overbrace{\vdots}^{i}=\lambda .
\end{aligned}
$$

This implies in particular that $\lambda$ is a morphism in $\mathscr{L}(\mathscr{C})$ from $(C, \sigma)$ to $\mathbb{1}_{\mathscr{L}(\mathscr{C})}$, since $\omega$ and $\Lambda$ are morphisms in $\mathscr{L}(\mathscr{C})$. 
Thirdly, $\lambda$ is a left cointegral for the Hopf algebra $(C, \sigma)$ in $\mathscr{E}(\mathscr{C})$. Indeed, using the description of the coproduct $\Delta$ and the unit $u$-items (a) and (d) on page $10-$ we obtain

$$
\begin{aligned}
\left(\operatorname{id}_{C} \otimes \lambda\right) \Delta & =\sum_{i, k, \ell, n \in I} \operatorname{dim}_{r}(k) \\
& =\sum_{i, k, \ell \in I} \operatorname{dim}_{r}(k) \\
& =\sum_{i, k, \ell \in I} \operatorname{dim}_{r}(k) \sum_{i, k, \ell \in I} \operatorname{dim}_{r}(i) \\
& =\sum_{i, \ell \in I} \operatorname{dim}_{r}(i) \\
& =u \lambda .
\end{aligned}
$$

Since $\omega(\Lambda \otimes \Lambda)=\lambda \Lambda=\operatorname{dim}_{r}(\mathbb{1})=1 \in \mathbb{k}$ is invertible, we conclude by Lemma 3.1 that $\omega$ is nondegenerate. Hence $\mathscr{L}(\mathscr{C})$ is modular.

Finally, let us prove that $\Lambda$ is a two-sided integral of $(C, \sigma)$ which is invariant under the antipode. The last part of Lemma 3.1 gives that $\Lambda$ is a right integral of $(C, \sigma)$. Using the description of the antipode $S$ of $(C, \sigma)$ in item (e) on page 11, we obtain

$$
S \Lambda=\sum_{j, k, \ell \in I} \operatorname{dim}_{r}(j)
$$




$$
\begin{aligned}
& =\sum_{\ell \in I} \operatorname{dim}_{r}\left(\ell^{*}\right) \\
& =\sum_{\ell \in I} \operatorname{dim}_{r}(\ell) \underbrace{}_{j} \text { by Lemma 4.1(d) } \\
& =\Lambda .
\end{aligned}
$$

Hence $\Lambda$ is $S$-invariant. This implies in particular that $\Lambda$, being a right integral, is also a left integral. Hence $\Lambda$ is an $S$-invariant (two-sided) integral.

4E. Proof of Theorem 2.2. Consider the Hopf monad $Z$ of Section 4B. Recall from [Bruguières and Virelizier 2007] that the monad $Z$ is said to be semisimple if any $Z$-module is a $Z$-linear retract of a free $Z$-module, that is, of $\left(Z(X), \mu_{X}\right)$ for some object $X$ of $\mathscr{C}$. Since the isomorphism $\Phi: \mathscr{C}^{Z} \rightarrow \mathscr{L}(\mathscr{C})$ defined in (8) sends the free $Z$-module $\left(Z(X), \mu_{X}\right)$ to the free half braiding $\Phi\left(Z(X), \mu_{X}\right)=\mathscr{F}(X)$, we need to prove that $\operatorname{dim}(\mathscr{C})$ is invertible if and only if $Z$ is semisimple. Now Theorem 6.5 of [Bruguières and Virelizier 2007] provides an analogue of Maschke's semisimplicity criterion for Hopf monads: the Hopf monad $Z$ is semisimple if and only if there exists a morphism $\alpha: \mathbb{1} \rightarrow Z(\mathbb{1})$ in $\mathscr{b}$ such that

$$
\mu_{\mathbb{1}} \alpha=\alpha Z_{0} \quad \text { and } \quad Z_{0} \alpha=1 .
$$

Let $\alpha: \mathbb{1} \rightarrow Z(\mathbb{1})=\bigoplus_{i \in I} i^{*} \otimes i$ be a morphism in $\mathscr{C}$. Since $\mathscr{C}$ is a fusion category, $\alpha$ decomposes uniquely as $\alpha=\sum_{i \in I} \alpha_{i} \widetilde{\cos }_{i}$ where $\alpha_{i} \in \mathbb{k}$. From the structural morphisms of the Hopf monad $Z$ (page 19), we obtain

$$
\alpha Z_{0}=\sum_{j, k \in I} \alpha_{k} \overbrace{k}^{j}
$$

and

$$
\mu_{\mathbb{1}} Z(\alpha)=\sum_{i, j, k \in I} \alpha_{i} \frac{\operatorname{dim}_{r}(k)}{\operatorname{dim}_{r}(i)}
$$

Thus, by duality, $\alpha Z_{0}=\mu_{\mathbb{1}} Z(\alpha)$ if and only if

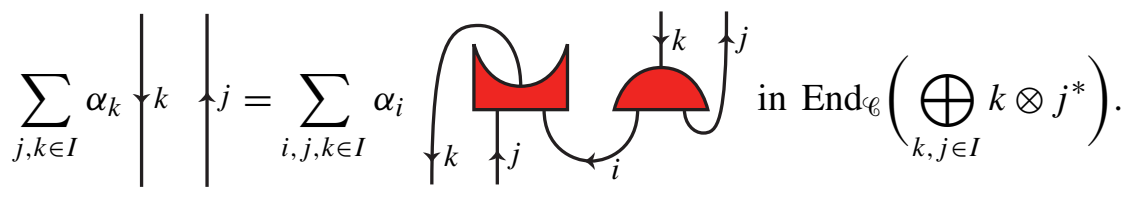


Now, for $j, k \in I$, by using Lemma 4.1(b),

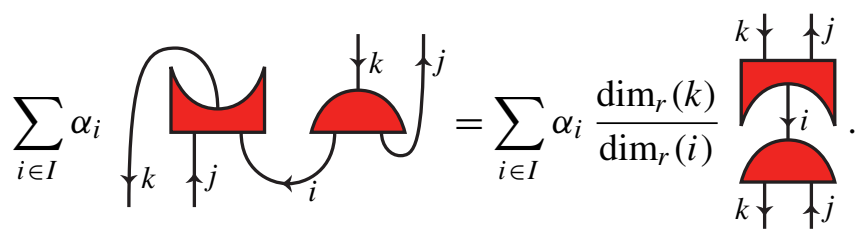

Therefore $\alpha Z_{0}=\mu_{\mathbb{1}} Z(\alpha)$ if and only if

$$
\alpha_{k} \operatorname{id}_{k \otimes j^{*}}=\sum_{i \in I} \alpha_{i} \underbrace{k \downarrow}_{k \nmid \uparrow_{j}} \in \operatorname{End}_{\mathscr{C}}\left(k \otimes j^{*}\right) \text { for all } k, j \in I .
$$

In particular, if $\alpha Z_{0}=\mu_{\mathbb{1}} Z(\alpha)$, then for any $i \in I$, setting $k=\mathbb{1}$ and $j=i^{*}$ we obtain $\alpha_{i}=\alpha_{\mathbb{1}} \operatorname{dim}_{r}(i)$. Conversely, if $\alpha_{i}=\alpha_{\mathbb{1}} \operatorname{dim}_{r}(i)$ for all $i \in I$, then (11) holds by Lemma 4.1(a), and so $\alpha Z_{0}=\mu_{\mathbb{1}} Z(\alpha)$. In conclusion, $\alpha Z_{0}=\mu_{\mathbb{1}} Z(\alpha)$ if and only if $\alpha=\alpha_{\mathbb{1}} \kappa$, where

$$
\kappa=\sum_{i \in I} \operatorname{dim}_{r}(i) \widetilde{\operatorname{cov}}_{i}: \mathbb{1} \rightarrow Z(\mathbb{1}) .
$$

In that case,

$$
Z_{0} \alpha=\alpha_{\mathbb{1}} Z_{0} \kappa=\sum_{i \in I} \operatorname{dim}_{r}(i) Z_{0} \widetilde{\operatorname{cev}}_{i}=\alpha_{\mathbb{1}} \sum_{i \in I} \operatorname{dim}_{r}(i) \operatorname{dim}_{l}(i)=\alpha_{\mathbb{1}} \operatorname{dim}(\mathscr{C}) .
$$

Hence there exists $\alpha$ satisfying (10) if and only if $\operatorname{dim}(\mathscr{C})$ is invertible in $\mathbb{k}$. This concludes the proof of Theorem 2.2.

4F. Proof of Corollary 2.3. Let $\mathscr{A}$ be an abelian category. If $\mathscr{A}$ is semisimple (see Section 2), then every object of $\mathscr{A}$ is projective ${ }^{2}$. The converse is true if in addition we assume that all objects of $\mathscr{A}$ have finite length ${ }^{3}$.

Assume $\mathbb{k}$ is a field and let $\mathscr{C}$ be a pivotal fusion category over $\mathbb{k}$. Then $\mathscr{C}$ is abelian semisimple and its objects have finite length. The center $\mathscr{L}(\mathscr{C})$ of $\mathscr{C}$ is then an abelian category and the forgetful functor $\mathscr{U}: \mathscr{L}(\mathscr{C}) \rightarrow \mathscr{C}$ is $\mathbb{k}$-linear, faithful, and exact. This implies that all objects of $\mathscr{L}(\mathscr{C})$ have finite length and the Hom spaces in $\mathscr{L}(\mathscr{C})$ are finite-dimensional. As a result, $\mathscr{L}(\mathscr{C})$ is semisimple if and only if all of its objects are projective.

\footnotetext{
${ }^{2}$ An object $P$ of $\mathscr{A}$ is projective if the functor $\operatorname{Hom}_{\mathscr{A}}(P,-): \mathscr{A} \rightarrow \mathrm{Ab}$ is exact, where $\mathrm{Ab}$ is the category of abelian groups.

${ }^{3}$ An object $A$ of $\mathscr{A}$ has finite length if there exists a finite sequence of subobjects $A=X_{0} \supsetneq X_{1} \supsetneq$ $\cdots \supsetneq X_{n}=\mathbf{0}$ such that each quotient $X_{i} / X_{i+1}$ is simple.
} 
We identify $\mathscr{L}(\mathscr{C})$ with the category $\mathscr{C}^{Z}$ of $Z$-modules via the isomorphism (8). Recall from the proof of Theorem 2.2 (see the beginning of Section $4 \mathrm{E}$ ) that the monad $Z$ is semisimple if and only if $\operatorname{dim}(\mathscr{C})$ is invertible in $\mathbb{k}$. The following lemma relates the notions of semisimplicity for monads and for categories.

Lemma 4.2. Let $\mathscr{b}$ be an abelian category and $T$ be a right exact monad on $\mathscr{b}$, so that $\mathscr{C}^{T}$ is abelian and the forgetful functor $U_{T}: \mathscr{C}^{T} \rightarrow \mathscr{C}$ is exact.

(a) If all the $T$-modules are projective, then $T$ is semisimple.

(b) If $T$ is semisimple and all the objects of $\mathscr{C}$ are projective, then all the $T$-modules are projective.

(c) If the objects of $\mathscr{C}$ have finite length, then the same holds in $\mathscr{C}^{T}$. If in addition $\mathscr{C}$ has finitely many isomorphy classes of simple objects, then so does $\mathscr{C}^{T}$.

Proof. Let us prove assertion (a). Denote by $F_{T}: \mathscr{C} \rightarrow \mathscr{C}^{T}$ the free module functor (see Section 4A). Let $(M, r)$ be a $T$-module. The action $r$ defines an epimorphism $F_{T}(M) \rightarrow(M, r)$ in $\mathscr{C}^{T}$. In particular, if $(M, r)$ is projective, it is a retract of $F_{T}(M)$. Therefore if all the $T$-modules are projective, the monad $T$ is semisimple.

Let us prove assertion (b). Note that if $X$ is a projective object of $\mathscr{C}$, then $F_{T}(X)$ is a projective $T$-module. Indeed,

$$
\operatorname{Hom}_{\mathscr{C} T}\left(F_{T}(X), ?\right) \simeq \operatorname{Hom}_{\mathscr{C}}\left(X, U_{T}\right)
$$

by adjunction, and $\operatorname{Hom}_{\mathscr{C}}\left(X, U_{T}\right)$ is an exact functor when $X$ is projective. In particular, if all objects are projective in $\mathscr{C}$ then all free $T$-modules are projective. If in addition $T$ is semisimple, then any $T$-module, being a retract of a free $T$-module, is projective.

Finally, let us prove assertion (c). The first part results from the fact that $U_{T}$ is faithful exact. Now if $S$ is a simple object of $\mathscr{C}^{T}$ and $\Sigma$ is a simple subobject of $U_{T}(S)$, then by adjunction the inclusion $\Sigma \subset U_{T}(S)$ defines a nonzero morphism $F_{T}(\Sigma) \rightarrow S$, which is an epimorphism because $S$ is simple. This proves the second part of assertion (c), because under the assumptions made there are finitely many possibilities for $\Sigma$, and each $F_{T}(\Sigma)$ has finitely many simple quotients.

Assertion (a) of Corollary 2.3 results immediately from the first two assertions of Lemma 4.2.

Let us prove assertion (b). A fusion category over a field is semisimple. Now assume $\mathbb{k}$ is algebraically closed. By assertion (a), we need to show that if $\mathscr{L}(\mathscr{C})$ is semisimple, then it is a fusion category. Assume $\mathscr{E}(\mathscr{C})$ is semisimple. Since $\mathscr{C}$ is fusion, by the third assertion of Lemma 4.2 , the category $\mathscr{L}(\mathscr{C})$ has finitely many classes of simple objects and its objects have finite length. So each object of $\mathscr{L}(\mathscr{C})$ is a finite direct sum of simple objects. Since the unit object of $\mathscr{L}(\mathscr{C})$ is scalar and any simple object $S$ of $\mathscr{L}(\mathscr{C})$ is scalar (because $\operatorname{End}(S)$ is a finite extension of $\mathbb{k}$ ), we obtain that $\mathscr{L}(\mathscr{C})$ is a fusion category. This proves Corollary 2.3. 


\section{References}

[Balsam 2010] B. Balsam, "Turaev-Viro invariants as an extended TQFT II", preprint, 2010. arXiv 1010.1222

[Bruguières and Virelizier 2007] A. Bruguières and A. Virelizier, "Hopf monads", Adv. Math. 215:2 (2007), 679-733. MR 2009b:18006 Zbl 1168.18002

[Bruguières and Virelizier 2008] A. Bruguières and A. Virelizier, "Categorical centers and Reshetikhin-Turaev invariants", Acta Math. Vietnam. 33:3 (2008), 255-277. MR 2010h:57017 Zbl 1226. 57018

[Bruguières and Virelizier 2012] A. Bruguières and A. Virelizier, "Quantum double of Hopf monads and categorical centers", Trans. Amer. Math. Soc. 364:3 (2012), 1225-1279. MR 2869176 Zbl 06024127

[Bruguières et al. 2011] A. Bruguières, S. Lack, and A. Virelizier, "Hopf monads on monoidal categories", Adv. Math. 227:2 (2011), 745-800. MR 2012h:18003 Zbl 1233.18002

[Etingof et al. 2005] P. Etingof, D. Nikshych, and V. Ostrik, "On fusion categories", Ann. of Math.

(2) 162:2 (2005), 581-642. MR 2006m:16051 Zbl 1125.16025

[Joyal and Street 1991] A. Joyal and R. Street, "Tortile Yang-Baxter operators in tensor categories", J. Pure Appl. Algebra 71:1 (1991), 43-51. MR 92e:18006 Zbl 0726.18004

[Lyubashenko 1995] V. V. Lyubashenko, "Invariants of 3-manifolds and projective representations of mapping class groups via quantum groups at roots of unity", Comm. Math. Phys. 172:3 (1995), 467-516. MR 97c:57018 Zbl 0844.57016

[Mac Lane 1998] S. Mac Lane, Categories for the working mathematician, 2nd ed., Graduate Texts in Mathematics 5, Springer, New York, 1998. MR 2001j:18001 Zbl 0906.18001

[Majid 1991] S. Majid, "Representations, duals and quantum doubles of monoidal categories", Rend. Circ. Mat. Palermo (2) Suppl. 26 (1991), 197-206. MR 93c:18008 Zbl 0762.18005

[Majid 1995] S. Majid, Foundations of quantum group theory, Cambridge University Press, Cambridge, 1995. MR 97g:17016 Zbl 0857.17009

[Müger 2003] M. Müger, "From subfactors to categories and topology, II: The quantum double of tensor categories and subfactors", J. Pure Appl. Algebra 180:1-2 (2003), 159-219. MR 2004f:18014 Zbl 1033.18003

[Turaev 1994] V. G. Turaev, Quantum invariants of knots and 3-manifolds, De Gruyter Studies in Mathematics 18, de Gruyter, Berlin, 1994. MR 95k:57014 Zbl 0812.57003

[Turaev and Virelizier 2010] V. G. Turaev and A. Virelizier, "On two approaches to 3-dimensional TQFTs", preprint, 2010. arXiv 1006.3501 
Received March 21, 2012. Revised August 28, 2012.

Alain BruguiÈres

DÉPARTEMENT DE MATHÉMATIQUES

UNIVERSITÉ MONTPELLIER II

CASE COURRIER 051

Place Eugène Bataillon

34095 Montpellier Cedex 5

FRANCE

bruguier@math.univ-montp2.fr

ALEXIS VIRELIZIER

DÉPARTEMENT DE MATHÉMATIQUES

UNIVERSITÉ LILLE 1

59655 Villeneuve D'AsCQ

FRANCE

alexis.virelizier@math.univ-lille1.fr 


\title{
PACIFIC JOURNAL OF MATHEMATICS
}

\author{
msp.org/pjm
}

Founded in 1951 by E. F. Beckenbach (1906-1982) and F. Wolf (1904-1989)

\section{EDITORS}

V. S. Varadarajan (Managing Editor)

Department of Mathematics

University of California

Los Angeles, CA 90095-1555

pacific@math.ucla.edu

Paul Balmer

Department of Mathematics

University of California

Los Angeles, CA 90095-1555

balmer@math.ucla.edu

Daryl Cooper

Department of Mathematics

University of California

Santa Barbara, CA 93106-3080 cooper@math.ucsb.edu

Jiang-Hua $\mathrm{Lu}$

Department of Mathematics

The University of Hong Kong

Pokfulam Rd., Hong Kong jhlu@maths.hku.hk
Don Blasius

Department of Mathematics University of California

Los Angeles, CA 90095-1555

blasius@math.ucla.edu

Robert Finn

Department of Mathematics Stanford University

Stanford, CA 94305-2125

finn@math.stanford.edu

Sorin Popa

Department of Mathematics

University of California

Los Angeles, CA 90095-1555

popa@math.ucla.edu

Paul Yang

Department of Mathematics

Princeton University

Princeton NJ 08544-1000

yang@math.princeton.edu

\section{PRODUCTION}

Silvio Levy, Scientific Editor, production@msp.org

\section{SUPPORTING INSTITUTIONS}

ACADEMIA SINICA, TAIPEI

CALIFORNIA INST. OF TECHNOLOGY

INST. DE MATEMÁTICA PURA E APLICADA

KEIO UNIVERSITY

MATH. SCIENCES RESEARCH INSTITUTE

NEW MEXICO STATE UNIV.

OREGON STATE UNIV.

\author{
STANFORD UNIVERSITY \\ UNIV. OF BRITISH COLUMBIA \\ UNIV. OF CALIFORNIA, BERKELEY \\ UNIV. OF CALIFORNIA, DAVIS \\ UNIV. OF CALIFORNIA, LOS ANGELES \\ UNIV. OF CALIFORNIA, RIVERSIDE \\ UNIV. OF CALIFORNIA, SAN DIEGO \\ UNIV. OF CALIF., SANTA BARBARA
}

\author{
Vyjayanthi Chari \\ Department of Mathematics \\ University of California \\ Riverside, CA 92521-0135 \\ chari@math.ucr.edu \\ Kefeng Liu \\ Department of Mathematics \\ University of California \\ Los Angeles, CA 90095-1555 \\ liu@math.ucla.edu \\ Jie Qing \\ Department of Mathematics \\ University of California \\ Santa Cruz, CA 95064 \\ qing@cats.ucsc.edu
}

These supporting institutions contribute to the cost of publication of this Journal, but they are not owners or publishers and have no responsibility for its contents or policies.

See inside back cover or msp.org/pjm for submission instructions.

The subscription price for 2013 is US \$400/year for the electronic version, and \$485/year for print and electronic.

Subscriptions, requests for back issues and changes of subscribers address should be sent to Pacific Journal of Mathematics, P.O. Box 4163, Berkeley, CA 94704-0163, U.S.A. The Pacific Journal of Mathematics is indexed by Mathematical Reviews, Zentralblatt MATH, PASCAL CNRS Index, Referativnyi Zhurnal, Current Mathematical Publications and the Science Citation Index.

The Pacific Journal of Mathematics (ISSN 0030-8730) at the University of California, c/o Department of Mathematics, 798 Evans Hall \#3840, Berkeley, CA 94720-3840, is published monthly except July and August. Periodical rate postage paid at Berkeley, CA 94704, and additional mailing offices. POSTMASTER: send address changes to Pacific Journal of Mathematics, P.O. Box 4163, Berkeley, CA 94704-0163.

PJM peer review and production are managed by EditFLOW ${ }^{\circledR}$ from Mathematical Sciences Publishers.

PUBLISHED BY

mathematical sciences publishers

nonprofit scientific publishing

http://msp.org/

(C) 2013 Mathematical Sciences Publishers 


\section{PACIFIC JOURNAL OF MATHEMATICS}

Volume $264 \quad$ No. $1 \quad$ July 2013

On the center of fusion categories

ALAIN BRUGUIÈRES and ALEXIS VIRELIZIER

Connected quandles associated with pointed abelian groups

W. EdWin Clark, MoHamed ElHamdadi, Xiang-DONG HoU,

MASAHICO SAITO and TIMOTHY YEATMAN

Entropy and lowest eigenvalue on evolving manifolds

HongXin GuO, ROBERT PHILIPOWSKI and ANTON THALMAIER

Poles of certain residual Eisenstein series of classical groups

DIHUA JIANG, BAIYING LIU and LEI ZHANG

Harmonic maps on domains with piecewise Lipschitz continuous metrics 125

HAIGANG LI and CHANGYOU WANG

$q$-hypergeometric double sums as mock theta functions

JEREMY LOVEJOY and ROBERT OSBURN

Monic representations and Gorenstein-projective modules

XIU-HuA LUO and PU ZHANG

Helicoidal flat surfaces in hyperbolic 3-space

Antonio Martínez, JoÃo Paulo dos Santos and Keti

TENENBLAT

On a Galois connection between the subfield lattice and the multiplicative subgroup lattice

JOHN K. MCVEY

Some characterizations of Campanato spaces via commutators on Morrey 221 spaces

Shaoguang Shi and Shanzhen Lu

The Siegel-Weil formula for unitary groups 